\title{
Dynamical analysis in exponential RED algorithm with communication delay
}

\author{
Changjin $\mathrm{Xu}^{1 *}$ and Peiluan $\mathrm{Li}^{2}$
}

\footnotetext{
"Correspondence: xcj403@126.com

'Guizhou Key Laboratory of

Economics System Simulation,

Guizhou University of Finance and

Economics, Guiyang, 550004,

P.R. China

Full list of author information is

available at the end of the article
}

\begin{abstract}
In this paper, an exponential RED algorithm with communication delay is considered. By choosing the delay as a bifurcation parameter, we demonstrate that a Hopf bifurcation would occur when the delay exceeds a critical value. Some explicit formulas are worked out for determining the stability and the direction of the bifurcated periodic solutions by using the normal form theory and center manifold theory. Finally, numerical simulations supporting the theoretical analysis are provided.
\end{abstract}

Keywords: exponential RED; stability; Hopf bifurcation; congestion control; periodic solution

\section{Introduction}

It is well known that Internet applications, such as the world wide web, file transfer, Usenet news and remote login, are delivered via the Transmission Control Protocol (TCP). With a spectacular growth of Internet applications, congestion control has become a subject of intense research activity. An uncontrolled network may suffer from severe congestion, which can cause high packet loss rates and increasing delays, the upper formation application systems performance drop, and it can even break the whole system by causing congestion collapse (or Internet meltdown) [1]. Thus congestion control is one of the critical issues for the efficient operation of the Internet. Many researchers have investigated the congestion control problems of the Internet and a lot of excellent and interesting results have been obtained, for example, Raina and Heckmann [2] investigated the stability properties of the congestion avoidance phase of TCP with drop tail. Guo et al. [3] investigated the stability of an exponential RED model with heterogeneous delays. Liu et al. [1] studied the bifurcation and chaotic behavior of the Transmission Control Protocol (TCP) and User Datagram Protocol (UDP) network with Random Early Detection (RED) queue management. Xu et al. [4] studied the Local Hopf bifurcation and global existence of periodic solutions of the following TCP system:

$$
\frac{d x(t)}{d t}=x(t-\tau)\left[\frac{M(1-p(x(t-\tau)))}{N x(t)}-\frac{x(t) p(x(t-\tau))}{2 M}\right],
$$

where $M, N$ are two positive constants, $p(y)$ is an increasing non-negative continuous function in $(0,+\infty)$ and $\tau$ is the round-trip propagation delay for each of the TCP connections. Guo et al. [5] considered the stability and Hopf bifurcation of the following conges-

(c) $2016 \mathrm{Xu}$ and Li. This article is distributed under the terms of the Creative Commons Attribution 4.0 International License (http://creativecommons.org/licenses/by/4.0/), which permits unrestricted use, distribution, and reproduction in any medium, provided you give appropriate credit to the original author(s) and the source, provide a link to the Creative Commons license, and indicate if changes were made. 
tion control model with two communication delays:

$$
\left\{\begin{array}{l}
\dot{x}_{1}(t)=k_{1} x_{1}\left(t-\tau_{1}\right)\left[\frac{\alpha}{x_{1}(t)}-\beta x_{1}(t) p\left(t-\tau_{1}\right)\right] \\
\dot{x}_{2}(t)=k_{2} x_{2}\left(t-\tau_{2}\right)\left[\frac{\alpha}{x_{2}(t)}-\beta x_{2}(t) p\left(t-\tau_{2}\right)\right] \\
\dot{p}(t)=\gamma p(t)\left[x_{1}\left(t-\tau_{1}\right)+x_{2}\left(t-\tau_{2}\right)-c\right]
\end{array}\right.
$$

where $x_{i}(t)$ is the rate at which source $i$ transmits data at time $t, \alpha$ and $\beta$ are positive real numbers, $p(t)$ is the loss probability function, $\tau_{i}$ is the round-trip delay for source $i, c$ is the link capacity, $k$ and $\gamma$ are positive gain parameter, $i=1,2$. For more related work on congestion control, see [6-12].

Based on [7-9], Guo et al. [13] recently studied the following exponential RED algorithm coupled with TCP-Reno with a single source and with two sources:

$$
\left\{\begin{array}{l}
\dot{x}_{1}(t)=k_{1} x_{1}(t-\tau)\left[\frac{1-p(t)}{\alpha^{2} x_{1}(t)}-\beta_{1} x_{1}(t) p(t)\right], \\
\dot{x}_{2}(t)=k_{2} x_{2}(t-\tau)\left[\frac{1-p(t)}{\alpha^{2} x_{2}(t)}-\beta_{2} x_{2}(t) p(t)\right], \\
\dot{p}(t)=k_{3} p(t)\left[x_{1}(t-\tau)+x_{2}(t-\tau)-c\right],
\end{array}\right.
$$

where $\dot{x}_{i}(t)=\frac{d x}{d t}(i=1,2,3)$ represent the transmission rate of the $i$ source per second at time $t, \tau, k_{i}(i=1,2,3)$ and $\beta_{j}(j=1,2)$ are the round-trip time (RTT), the positive gain parameter, and the decrease factor of the source $i$, respectively. By choosing the delay as a bifurcation parameter, Guo et al. [13] obtained the necessary and sufficient conditions for the existence of Hopf bifurcation and a formula for determining the direction of the Hopf bifurcation and the stability of bifurcating periodic solutions.

Motivated by [13] and considering that, when the number of sources is large, the simplified model can reflect the really exponential RED algorithm more closely, in this paper, we consider an exponential RED algorithm coupled with TCP-Reno with a single source and with three sources, then we have the following system:

$$
\left\{\begin{array}{l}
\dot{x}_{1}(t)=k_{1} x_{1}(t-\tau)\left[\frac{1-p(t)}{\alpha^{2} x_{1}(t)}-\beta_{1} x_{1}(t) p(t)\right] \\
\dot{x}_{2}(t)=k_{2} x_{2}(t-\tau)\left[\frac{1-p(t)}{\alpha^{2} x_{2}(t)}-\beta_{2} x_{2}(t) p(t)\right] \\
\dot{x}_{3}(t)=k_{3} x_{3}(t-\tau)\left[\frac{1-p(t)}{\alpha^{2} x_{3}(t)}-\beta_{3} x_{3}(t) p(t)\right] \\
\dot{p}(t)=k_{4} p(t)\left[x_{1}(t-\tau)+x_{2}(t-\tau)+x_{3}(t-\tau)-c\right] .
\end{array}\right.
$$

The purpose of this paper is to discuss the stability and the properties of the Hopf bifurcation of model (1.4). This paper is organized as follows. In Section 2, the stability of the equilibrium and the existence of a Hopf bifurcation at the equilibrium are studied. In Section 3, the direction of the Hopf bifurcation and the stability and periodic of bifurcating periodic solutions on the center manifold are determined. In Section 4, numerical simulations are carried out to illustrate the validity of the main results. Some main conclusions are drawn in Section 5.

\section{Stability of the equilibrium and local Hopf bifurcations}

Let $E_{*}\left(x_{1}^{*}, x_{2}^{*}, x_{3}^{*}, p^{*}\right)$ be the non-zero equilibrium point of system (1.4), then we have

$$
x_{1}^{*}=\frac{1}{\alpha} \sqrt{\frac{1-p^{*}}{\beta_{1} p^{*}}}, \quad x_{2}^{*}=\frac{1}{\alpha} \sqrt{\frac{1-p^{*}}{\beta_{2} p^{*}}}, \quad x_{3}^{*}=\frac{1}{\alpha} \sqrt{\frac{1-p^{*}}{\beta_{3} p^{*}}},
$$


where $p^{*}$ satisfies the following equation:

$$
\sqrt{\frac{1-p^{*}}{\beta_{1} p^{*}}}+\sqrt{\frac{1-p^{*}}{\beta_{2} p^{*}}}+\sqrt{\frac{1-p^{*}}{\beta_{3} p^{*}}}=c \alpha .
$$

Let

$$
\begin{array}{ll}
y_{1}(t)=x_{1}(t)-x_{1}^{*}, & y_{2}(t)=x_{2}(t)-x_{2}^{*}, \\
y_{3}(t)=x_{3}(t)-x_{3}^{*}, & y_{4}(t)=p(t)-p^{*} .
\end{array}
$$

Substituting (2.2) into (1.4), we obtain the following linearized system:

$$
\left\{\begin{array}{l}
\dot{y}_{1}(t)=a_{1} y_{1}(t)+a_{2} y_{4}(t), \\
\dot{y}_{2}(t)=b_{1} y_{2}(t)+b_{2} y_{4}(t), \\
\dot{y}_{3}(t)=c_{1} y_{3}(t)+c_{2} y_{4}(t), \\
\dot{y}_{4}(t)=d_{1} y_{1}(t-\tau)+d_{1} y_{2}(t-\tau)+d_{1} y_{3}(t-\tau)
\end{array}\right.
$$

where

$$
\begin{aligned}
& a_{1}=-2 k_{1} \beta_{1} p^{*} x_{1}^{*}, \quad b_{1}=-2 k_{2} \beta_{2} p^{*} x_{2}^{*}, \quad c_{1}=-2 k_{3} \beta_{3} p^{*} x_{3}^{*}, \quad d_{1}=k_{3} p^{*}, \\
& a_{2}=-\frac{k_{1} \beta_{1}\left(x_{1}^{*}\right)^{2}}{1-p^{*}}, \quad b_{2}=-\frac{k_{2} \beta_{2}\left(x_{2}^{*}\right)^{2}}{1-p^{*}}, \quad c_{2}=-\frac{k_{3} \beta_{3}\left(x_{3}^{*}\right)^{2}}{1-p^{*}} \text {. }
\end{aligned}
$$

Then the associated characteristic equation of (2.3) is

$$
\operatorname{det}\left(\begin{array}{cccc}
\lambda-a_{1} & 0 & 0 & -a_{2} \\
0 & \lambda-b_{1} & 0 & -b_{2} \\
0 & 0 & \lambda-c_{1} & -c_{2} \\
-d_{1} e^{-\lambda \tau} & -d_{1} e^{-\lambda \tau} & -d_{1} e^{-\lambda \tau} & \lambda
\end{array}\right)=0
$$

Then we obtain the following fourth degree exponential polynomial equation:

$$
\lambda^{4}+m_{1} \lambda^{3}+m_{2} \lambda^{2}+m_{3} \lambda+\left(n_{1} \lambda^{2}+n_{2} \lambda+n_{3}\right) e^{-\lambda \tau}=0,
$$

where

$$
\begin{aligned}
& m_{1}=-\left(a_{1}+b_{1}+c_{1}\right), \quad m_{2}=a_{1} b_{1}+a_{1} c_{1}+b_{1} c_{1}, \quad m_{3}=-a_{1} b_{1} c_{1}, \\
& n_{1}=-\left(b_{2} d_{2}+c_{2} d_{3}+a_{2} d_{1}\right), \quad n_{2}=b_{2} d_{2}\left(a_{1}+c_{1}\right)+c_{2} d_{3}\left(a_{1}+b_{1}\right)+a_{2} d_{1}\left(b_{1}+c_{1}\right), \\
& n_{3}=-\left(a_{1} b_{2} c_{1} d_{2}+a_{1} b_{1} c_{2} d_{3}+a_{2} b_{1} c_{1} d_{1}\right) .
\end{aligned}
$$

Let $\lambda=i \omega_{0}, \tau=\tau_{0}$, and substitute this into (2.5), for the sake of simplicity, denote $\omega_{0}$ and $\tau_{0}$ by $\omega, \tau$, respectively, Separating the real and imaginary parts, we have

$$
\begin{aligned}
& \left(n_{3}-n_{1} \omega^{2}\right) \cos \omega \tau+n_{2} \omega \sin \omega \tau=m_{2} \omega^{2}-\omega^{4}, \\
& n_{2} \omega \cos \omega \tau-\left(n_{3}-n_{1} \omega^{2}\right) \sin \omega \tau=m_{1} \omega^{3}-m_{3} \omega .
\end{aligned}
$$


Taking the square on both sides of (2.6) and (2.7) and summing them up, we obtain

$$
\left(n_{3}-n_{1} \omega^{2}\right)^{2}+\left(n_{2} \omega\right)^{2}=\left(m_{2} \omega^{2}-\omega^{4}\right)^{2}+\left(m_{1} \omega^{3}-m_{3} \omega\right)^{2}
$$

i.e.,

$$
\omega^{8}+\left(m_{1}-2 n_{2}\right) \omega^{6}+\left(n_{2}-2 m_{1} m_{3}-n_{1}^{2}\right) \omega^{4}+\left(m_{3}+2 n_{1} n_{3}-n_{2}^{2}\right) \omega^{2}-n_{3}^{2}=0 .
$$

Let $p=m_{1}-2 n_{2}, q=n_{2}-2 m_{1} m_{3}-n_{1}^{2}, u=m_{3}+2 n_{1} n_{3}-n_{2}^{2}, v=-n_{3}^{2}, z=\omega^{2}$. Then equation (2.8) becomes

$$
z^{4}+p z^{3}+q z^{2}+u z+v=0
$$

Let

$$
l(z)=z^{4}+p z^{3}+q z^{2}+u z+v .
$$

If the coefficients $k_{i}(i=1,2,3,4), \alpha, \beta_{j}(j=1,2,3)$ of system (1.4) are given, it is easy to use computer to calculate the roots of (2.9). Since $\lim _{z \rightarrow \infty} l(z)=+\infty$ and $v<0$, we can conclude that (2.9) has at least one positive real root.

Without loss of generality, we assume that (2.9) has four positive roots, denoted by $z_{1}$, $z_{2}, z_{3}, z_{4}$, respectively. Then (2.8) has four positive roots

$$
\omega_{1}=\sqrt{z_{1}}, \quad \omega_{2}=\sqrt{z_{2}}, \quad \omega_{3}=\sqrt{z_{3}}, \quad \omega_{4}=\sqrt{z_{4}} .
$$

By (2.6) and (2.7) we have

$$
\tau_{k}^{(j)}=\frac{1}{\omega_{k}}\left[\arccos \frac{\left(m_{2} \omega_{k}^{2}-m^{4}\right)\left(n_{3}-n_{1} \omega_{k}^{2}\right)+\left(m_{1} \omega_{k}^{3}-m_{3} \omega\right) n_{2} \omega}{\left(n_{3}-n_{1} \omega_{k}^{2}\right)^{2}+\left(n_{2} \omega\right)^{2}}+2 j \pi\right],
$$

where $k=1,2,3,4$ and $j=0,1,2, \ldots$. Then $\pm i \omega_{k}$ are a pair of purely imaginary roots of equation (2.5) with $\tau=\tau_{k}^{(j)}$. Obviously, the sequence $\left\{\tau_{k}^{(j)}\right\}_{j=0}^{+\infty}$ is increasing, and

$$
\lim _{j \rightarrow+\infty} \tau_{k}^{(j)}=+\infty, \quad k=1,2,3,4
$$

Then we can define

$$
\tau_{0}=\tau_{k 0}^{(0)}=\min _{1 \leq k \leq 4}\left\{\tau_{k}^{(0)}\right\}, \quad \omega_{0}=\omega_{k 0} .
$$

Note that, when $\tau=0$, (2.5) becomes

$$
\lambda^{4}+q_{1} \lambda^{3}+q_{2} \lambda^{2}+q_{3} \lambda+q_{4}=0,
$$

where

$$
\begin{aligned}
& q_{1}=-\left(a_{1}+b_{1}+c_{1}\right), \\
& q_{2}=\left(a_{1} b_{1}+a_{1} c_{1}+b_{1} c_{1}\right)-\left(b_{2} d_{2}+c_{2} d_{3}+a_{2} d_{1}\right),
\end{aligned}
$$




$$
\begin{aligned}
& q_{3}=b_{2} d_{2}\left(a_{1}+c_{1}\right)+c_{2} d_{3}\left(a_{1}+b_{1}\right)+a_{2} d_{1}\left(c_{1}+d_{1}\right)-a_{1} b_{1} c_{1} \\
& q_{4}=a_{1} b_{2} c_{1} d_{2}+a_{1} b_{1} c_{2} d_{3}+a_{2} b_{1} c_{1} d_{1} .
\end{aligned}
$$

A set of necessary and sufficient conditions for all roots of (2.13) to have a negative real part is given by the well-known Routh-Hurwitz criteria in the following form:

$$
q_{1}>0, \quad q_{1} q_{2}-q_{3}>0, \quad q_{1} q_{2} q_{3}-q_{3}^{2}-q_{1}^{2} q_{4}>0, \quad q_{4}>0
$$

Let $\lambda(\tau)=\alpha(\tau)+i \omega(\tau)$ be the root of equation (2.5) near $\tau=\tau_{k}^{(j)}$ satisfying $\alpha\left(\tau_{k}^{(j)}\right)=0$, $\omega\left(\tau_{k}^{(j)}\right)=\omega_{k}$. Then the following lemma holds.

Lemma 2.1 Suppose that $l^{\prime}\left(z_{k}\right) \neq 0$, where $l(z)$ is defined by $(2.10)$. If $\tau=\tau_{k}^{(j)}$, then $\pm i \omega_{k}$ are a pair of simple purely imaginary roots of equation (2.5). Moreover,

$$
\left[\frac{d(\operatorname{Re} \lambda(\tau))}{d \tau}\right]_{\tau=\tau_{k}^{(j)}} \neq 0
$$

and the sign of $\left[\frac{d(\operatorname{Re} \lambda(\tau))}{d \tau}\right]_{\tau=\tau_{k}^{(j)}}$ is consistent with that of $l^{\prime}\left(z_{k}\right)$.

Proof Let

$$
F(\lambda)=\lambda^{4}+m_{1} \lambda^{3}+m_{2} \lambda^{2}+m_{3} \lambda, \quad H(\lambda)=n_{1} \lambda^{2}+n_{2} \lambda+n_{3} .
$$

Then (2.5) can be written as

$$
F(\lambda)+H(\lambda) e^{-\lambda \tau}=0,
$$

which leads to

$$
F(\lambda) \overline{F(\lambda)}-H(\lambda) \overline{H(\lambda)}=0 .
$$

Thus, together with (2.9) and (2.10), we get

$$
l\left(\omega^{2}\right)=F(i \omega) \overline{F(i \omega)}-H(i \omega) \overline{H(i \omega)} .
$$

Differentiating both sides of equation (2.5) with respect to $\omega$, we obtain

$$
2 \omega l^{\prime}\left(\omega^{2}\right)=i\left[F^{\prime}(i \omega) \overline{F(i \omega)}-\overline{F^{\prime}(i \omega)} F(i \omega)-H^{\prime}(i \omega) \overline{H(i \omega)}+\overline{H^{\prime}(i \omega)} H(i \omega)\right] .
$$

If $i \omega_{k}$ is not simple, then $\omega_{k}$ must satisfy

$$
\left.\frac{d}{d \lambda}\left[F(\lambda)+H(\lambda) e^{-\lambda \tau_{k}^{(j)}}\right]\right|_{\lambda=i \omega_{k}}=0
$$

i.e.,

$$
F^{\prime}\left(i \omega_{k}\right)+H^{\prime}\left(i \omega_{k}\right) e^{-\omega_{k} \tau_{k}^{(j)}}-\tau_{k}^{(j)} H\left(i \omega_{k}\right) e^{-\omega_{k} \tau_{k}^{(j)}}=0 .
$$


By (2.16), we obtain

$$
\tau_{k}^{(j)}=\frac{H^{\prime}\left(i \omega_{k}\right)}{H\left(i \omega_{k}\right)}-\frac{F^{\prime}\left(i \omega_{k}\right)}{F\left(i \omega_{k}\right)} .
$$

Thus, by (2.17) and (2.19), we obtain

$$
\begin{aligned}
\operatorname{Im} \tau_{k}^{(j)} & =\operatorname{Im}\left\{\frac{H^{\prime}\left(i \omega_{k}\right)}{H\left(i \omega_{k}\right)}-\frac{F^{\prime}\left(i \omega_{k}\right)}{F\left(i \omega_{k}\right)}\right\}=\operatorname{Im}\left\{\frac{H^{\prime}\left(i \omega_{k}\right) \overline{H\left(i \omega_{k}\right)}}{H\left(i \omega_{k}\right) \overline{H\left(i \omega_{k}\right)}}-\frac{F^{\prime}\left(i \omega_{k}\right) \overline{F\left(i \omega_{k}\right)}}{F\left(i \omega_{k}\right) \overline{F\left(i \omega_{k}\right)}}\right\} \\
& =\operatorname{Im}\left\{\frac{H^{\prime}\left(i \omega_{k}\right) \overline{H\left(i \omega_{k}\right)}-F^{\prime}\left(i \omega_{k}\right) \overline{F\left(i \omega_{k}\right)}}{F\left(i \omega_{k}\right) \overline{F\left(i \omega_{k}\right)}}\right\}=\frac{\omega_{k} l^{\prime}\left(\omega_{k}^{2}\right)}{\left|F\left(i \omega_{k}\right)\right|^{2}} .
\end{aligned}
$$

Since $\tau_{k}^{(j)}$ is real, we have $l^{\prime}\left(z_{k}\right)=l^{\prime}\left(\omega_{k}^{2}\right)=0$, which is a contradiction to the condition $l^{\prime}\left(z_{k}\right) \neq 0$. This proves the first part of Lemma 2.1.

Taking the derivative of $\lambda$ with respect to $\tau$ in (2.5), it is easy to obtain

$$
\left[F^{\prime}(\lambda)+H^{\prime}(\lambda) e^{-\lambda \tau}-\tau H(\lambda) e^{-\lambda \tau}\right] \frac{d \lambda}{d \tau}-\lambda H(\lambda) e^{-\lambda \tau}=0
$$

which means

$$
\begin{aligned}
\frac{d \lambda(\tau)}{d \tau} & =\frac{\lambda H(\lambda)}{F^{\prime}(\lambda) e^{\lambda \tau}+H^{\prime}(\lambda)-\tau H(\lambda)} \\
& =\frac{\lambda H(\lambda)\left[\overline{F^{\prime}(\lambda)} e^{\lambda \tau}+\overline{H^{\prime}(\lambda)}-\tau \overline{H(\lambda)}\right]}{\left|F^{\prime}(\lambda) e^{\lambda \tau}+H^{\prime}(\lambda)-\tau H(\lambda)\right|^{2}} \\
& =\frac{\lambda\left[-\overline{F^{\prime}(\lambda)} F(\lambda)+\overline{H^{\prime}(\lambda)} H(\lambda)-\tau|H(\lambda)|^{2}\right]}{\left|F^{\prime}(\lambda) e^{\lambda \tau}+H^{\prime}(\lambda)-\tau H(\lambda)\right|^{2}} .
\end{aligned}
$$

It follows from this together with (2.19) that

$$
\begin{aligned}
& \left.\frac{d(\operatorname{Re} \lambda(\tau))}{d \tau}\right|_{\tau=\tau_{k}^{(j)}} \\
& =\left.\frac{\operatorname{Re}\left\{\lambda\left[-\overline{F^{\prime}(\lambda)} F(\lambda)+\overline{H^{\prime}(\lambda)} H(\lambda)-\tau|H(\lambda)|^{2}\right]\right\}}{\left|F^{\prime}(\lambda) e^{\lambda \tau}+H^{\prime}(\lambda)-\tau H(\lambda)\right|^{2}}\right|_{\tau=\tau_{k}^{(j)}} \\
& =\frac{i \omega_{k}\left[-\overline{F^{\prime}\left(i \omega_{k}\right)} F\left(i \omega_{k}\right)+\overline{H^{\prime}\left(i \omega_{k}\right)} H\left(i \omega_{k}\right)+F^{\prime}\left(i \omega_{k}\right) \overline{F\left(i \omega_{k}\right)}-H^{\prime}\left(i \omega_{k}\right) \overline{H\left(i \omega_{k}\right)}\right]}{2\left|F^{\prime}\left(i \omega_{k}\right) e^{i \tau_{k}^{(j)} \omega_{k}}+H^{\prime}\left(i \omega_{k}\right)-\tau_{k}^{(j)} H\left(i \omega_{k}\right)\right|^{2}} \\
& =\frac{\omega_{k}^{2} l^{\prime}\left(\omega_{k}^{2}\right)}{\left|F^{\prime}\left(i \omega_{k}\right) e^{i \tau_{k}^{(j)} \omega_{k}}+H^{\prime}\left(i \omega_{k}\right)-\tau_{k}^{(j)} H\left(i \omega_{k}\right)\right|^{2}} \\
& =\frac{\omega_{k}^{2} l^{\prime}\left(z_{k}\right)}{\left|F^{\prime}\left(i \omega_{k}\right) e^{i \tau_{k}^{(j)} \omega_{k}}+H^{\prime}\left(i \omega_{k}\right)-\tau_{k}^{(j)} H\left(i \omega_{k}\right)\right|^{2}} \neq 0 .
\end{aligned}
$$

Obviously, the sign of $\left.\frac{d(\operatorname{Re} \lambda(\tau))}{d \tau}\right|_{\tau=\tau_{k}^{(j)}}$ is determined by that of $l^{\prime}\left(z_{k}\right)$. This completes the proof.

In order to investigate the distribution of roots of the transcendental equation (2.5), the following lemma, which is stated in [14], is useful. 
Lemma 2.2 [14] For the transcendental equation

$$
\begin{aligned}
P\left(\lambda, e^{-\lambda \tau_{1}}, \ldots, e^{-\lambda \tau_{m}}\right)= & \lambda^{n}+p_{1}^{(0)} \lambda^{n-1}+\cdots+p_{n-1}^{(0)} \lambda+p_{n}^{(0)} \\
& +\left[p_{1}^{(1)} \lambda^{n-1}+\cdots+p_{n-1}^{(1)} \lambda+p_{n}^{(1)}\right] e^{-\lambda \tau_{1}}+\cdots \\
& +\left[p_{1}^{(m)} \lambda^{n-1}+\cdots+p_{n-1}^{(m)} \lambda+p_{n}^{(m)}\right] e^{-\lambda \tau_{m}}=0,
\end{aligned}
$$

as $\left(\tau_{1}, \tau_{2}, \tau_{3}, \ldots, \tau_{m}\right)$ vary, the sum of orders of the zeros of $P\left(\lambda, e^{-\lambda \tau_{1}}, \ldots, e^{-\lambda \tau_{m}}\right)$ in the open right half plane can change, and only a zero appears on or crosses the imaginary axis.

From Lemma 2.2, it is easy to obtain the following results.

Theorem 2.3 Let $\tau_{k}^{(j)}$ and $\tau_{0}$ be defined by (2.11) and (2.12), respectively.

(i) If (2.14) holds, the equilibrium $E_{*}\left(x_{1}^{*}, x_{2}^{*}, x_{3}^{*}, p^{*}\right)$ of system (1.4) is asymptotically stable for $\tau \in\left[0, \tau_{0}\right)$.

(ii) If (2.14) and $l^{\prime}\left(z_{k}\right)$ hold, then system (1.4) undergoes a Hopf bifurcation at the equilibrium $E_{*}\left(x_{1}^{*}, x_{2}^{*}, x_{3}^{*}, p^{*}\right)$ when $\tau=\tau_{k}^{(j)}$, i.e., system (1.4) has a branch of periodic solutions bifurcating from the equilibrium $E_{*}\left(x_{1}^{*}, x_{2}^{*}, x_{3}^{*}, p^{*}\right)$ near $\tau=\tau_{k}^{(j)}$.

\section{Direction and stability of the Hopf bifurcation}

In the previous section, we have obtained conditions for Hopf bifurcation to occur when $\tau=\tau_{k}^{(j)}$. In this section, we shall derive the explicit formulas for determining the direction, stability, and period of these periodic solutions bifurcating from the equilibrium $E_{*}\left(x_{1}^{*}, x_{2}^{*}, x_{3}^{*}, p^{*}\right)$ at these critical value of $\tau$, by using techniques from the normal form and center manifold theory [15], Throughout this section, we always assume that system (2.2) undergoes Hopf bifurcation at the equilibrium $E_{*}\left(x_{1}^{*}, x_{2}^{*}, x_{3}^{*}, p^{*}\right)$ for $\tau=\tau_{k}^{(j)}$, and then $\pm i \omega_{k}$ are corresponding purely imaginary roots of the characteristic equation at the equilibrium $E_{*}\left(x_{1}^{*}, x_{2}^{*}, x_{3}^{*}, p^{*}\right)$. The linear part of system (1.4) at $E_{*}\left(x_{1}^{*}, x_{2}^{*}, x_{3}^{*}, p^{*}\right)$ is given by

$$
\left\{\begin{array}{l}
\dot{y}_{1}(t)=a_{1} y_{1}(t)+a_{2} y_{4}(t), \\
\dot{y}_{2}(t)=b_{1} y_{2}(t)+b_{2} y_{4}(t), \\
\dot{y}_{3}(t)=c_{1} y_{3}(t)+c_{2} y_{4}(t) \\
\dot{y}_{4}(t)=d_{1} y_{1}(t-\tau)+d_{1} y_{2}(t-\tau)+d_{1} y_{3}(t-\tau)
\end{array}\right.
$$

and the non-linear part is given by

$$
f\left(\mu, u_{t}\right)=\left(f_{1}, f_{2}, f_{3}, f_{4}\right)^{T},
$$

where

$$
\begin{aligned}
f_{1}= & a_{3} y_{1}^{2}(t)+a_{4} y_{1}(t) y_{1}(t-\tau)+a_{5} y_{1}(t) y_{4}(t)+a_{6} y_{1}(t-\tau) y_{4}(t) \\
& +a_{7} y_{1}^{3}(t)+a_{8} y_{1}^{2}(t) y_{1}(t-\tau)+a_{9} y_{1}^{2}(t) y_{4}(t)+a_{10} y_{1}(t) y_{1}(t-\tau) y_{4}(t)+\text { h.o.t., } \\
f_{2}= & b_{3} y_{2}^{2}(t)+b_{4} y_{2}(t) y_{2}(t-\tau)+b_{5} y_{2}(t) y_{4}(t)+b_{6} y_{2}(t-\tau) y_{4}(t) \\
& +b_{7} y_{2}^{3}(t)+b_{8} y_{2}^{2}(t) y_{2}(t-\tau)+b_{9} y_{2}^{2}(t) y_{4}(t)+b_{10} y_{2}(t) y_{2}(t-\tau) y_{4}(t)+\text { h.o.t., } \\
f_{3}= & c_{3} y_{3}^{2}(t)+c_{4} y_{3}(t) y_{3}(t-\tau)+c_{5} y_{3}(t) y_{4}(t)+c_{6} y_{3}(t-\tau) y_{4}(t)
\end{aligned}
$$




$$
\begin{aligned}
& +c_{7} y_{3}^{3}(t)+c_{8} y_{3}^{2}(t) y_{3}(t-\tau)+c_{9} y_{3}^{2}(t) y_{4}(t)+c_{10} y_{3}(t) y_{3}(t-\tau) y_{4}(t)+\text { h.o.t., } \\
f_{4}= & d_{1} y_{1}(t-\tau) y_{4}(t)+d_{1} y_{2}(t-\tau) y_{4}(t)+d_{1} y_{3}(t-\tau) y_{4}(t),
\end{aligned}
$$

where

$$
\begin{array}{ll}
a_{3}=k_{1} \beta_{1} p^{*} x_{1}^{*}, & a_{4}=-2 k_{1} \beta_{1} p^{*}, \quad a_{5}=\frac{k_{1} \beta_{1} x_{1}^{*}\left(2 p^{*}-1\right)}{1-p^{*}}, \quad a_{6}=\frac{k_{1} \beta_{1} x_{1}^{*}}{1-p^{*}}, \\
a_{7}=-\frac{k_{1} \beta_{1} p^{*}}{x_{1}^{*}}, & a_{8}=\frac{k_{1} \beta_{1} p^{*}}{x_{1}^{*}}, \quad a_{9}=-\frac{k_{1} \beta_{1} p^{*}}{1-p^{*}}, \quad a_{10}=\frac{k_{1} \beta_{1}\left(2 p^{*}-1\right)}{1-p^{*}}, \\
b_{3}=k_{2} \beta_{2} p^{*} x_{2}^{*}, & b_{4}=-2 k_{2} \beta_{2} p^{*}, \quad b_{5}=\frac{k_{2} \beta_{2} x_{2}^{*}\left(2 p^{*}-1\right)}{1-p^{*}}, \\
b_{6}=\frac{k_{2} \beta_{2} x_{2}^{*}}{1-p^{*}}, & b_{7}=-\frac{k_{2} \beta_{2} p^{*}}{x_{2}^{*}}, \quad b_{8}=\frac{k_{2} \beta_{2} p^{*}}{x_{2}^{*}}, \\
b_{9}=-\frac{k_{2} \beta_{2} p^{*}}{1-p^{*}}, & b_{10}=\frac{k_{2} \beta_{2}\left(2 p^{*}-1\right)}{1-p^{*}}, \quad d_{1}=k_{4} .
\end{array}
$$

Set $\tau=\tau_{k}^{(j)}+\mu$ and denote

$$
\begin{aligned}
C^{k}[-\tau, 0]= & \left\{\varphi \mid \varphi:[-\tau, 0] \rightarrow R^{4}, \text { each component of } \varphi\right. \\
& \text { has } k \text { th order continuous derivative }\}
\end{aligned}
$$

For convenience, denote $C[-\tau, 0]$ by $C^{0}[-\tau, 0]$.

For $\varphi(\theta)=\left(\varphi_{1}(\theta), \varphi_{2}(\theta), \varphi_{3}(\theta), \varphi_{4}(\theta)\right)^{T} \in C\left([-\tau, 0], R^{4}\right)$, define a family of operators

$$
L_{\mu} \varphi=B \varphi(0)+B_{1} \varphi(-\tau)
$$

and

$$
G(\mu, \varphi)=\left(k_{1}, k_{2}, k_{3}, k_{4}\right)^{T},
$$

where

$$
\begin{aligned}
B= & \left(\begin{array}{cccc}
a_{1} & 0 & 0 & a_{2} \\
0 & b_{1} & 0 & b_{2} \\
0 & 0 & c_{1} & c_{2} \\
0 & 0 & 0 & 0
\end{array}\right), \quad B_{1}=\left(\begin{array}{cccc}
0 & 0 & 0 & 0 \\
0 & 0 & 0 & 0 \\
0 & 0 & 0 & 0 \\
d_{1} & d_{1} & d_{1} & 0
\end{array}\right) \\
k_{1}= & a_{3} \varphi_{1}^{2}(0)+a_{4} \varphi_{1}(0) \varphi_{1}(-\tau)+a_{5} \varphi_{1}(0) \varphi_{4}(0)+a_{6} \varphi_{1}(-\tau) \varphi_{4}(0) \\
& +a_{7} \varphi_{1}^{3}(0)+a_{8} \varphi_{1}^{2}(0) \varphi_{1}(-\tau)+a_{9} \varphi_{1}^{2}(t) \varphi_{4}(0)+a_{10} \varphi_{1}(t) \varphi_{1}(-\tau) \varphi_{4}(0)+o\left(\|\varphi\|^{4}\right), \\
k_{2}= & b_{3} \varphi_{2}^{2}(0)+b_{4} \varphi_{2}(0) \varphi_{2}(-\tau)+b_{5} \varphi_{2}(0) \varphi_{4}(0)+b_{6} \varphi_{2}(-\tau) y_{4}(0) \\
& +b_{7} \varphi_{2}^{3}(0)+b_{8} \varphi_{2}^{2}(0) \varphi_{2}(-\tau)+b_{9} \varphi_{2}^{2}(0) \varphi_{4}(0)+b_{10} \varphi_{2}(0) \varphi_{2}(-\tau) \varphi_{4}(0)+o\left(\|\varphi\|^{4}\right), \\
k_{3}= & c_{3} \varphi_{3}^{2}(0)+c_{4} \varphi_{3}(0) \varphi_{3}(-\tau)+c_{5} \varphi_{3}(0) \varphi_{4}(0)+c_{6} \varphi_{3}(t-\tau) \varphi_{4}(0) \\
& +c_{7} \varphi_{3}^{3}(0)+c_{8} \varphi_{3}^{2}(0) \varphi_{3}\left(0-\tau_{k}^{(j)}\right)+c_{9} \varphi_{3}^{2}(0) \varphi_{4}(0)+c_{10} \varphi_{3}(0) \varphi_{3}(-\tau) \varphi_{4}(0)+o\left(\|\varphi\|^{4}\right), \\
k_{4}= & d_{1} \varphi_{1}(-\tau) \varphi_{4}(0)+d_{1} \varphi_{2}(-\tau) \varphi_{4}(0)+d_{1} \varphi_{3}(-\tau) \varphi_{4}(0)
\end{aligned}
$$


and $L_{\mu}$ is a one-parameter family of bounded linear operators in $C\left([-\tau, 0], R^{4}\right) \rightarrow R^{4}$. By the Riesz representation theorem, there exists a matrix whose components are bounded variation functions $\eta(\theta, \mu)$ in $[-\tau, 0] \rightarrow R^{4^{2}}$, such that

$$
L_{\mu} \varphi=\int_{-\tau}^{0} d \eta(\theta, \mu) \varphi(\theta)
$$

In fact, choosing

$$
\eta(\theta, \mu)=B \delta(\theta)+B_{1} \delta(\theta+\tau),
$$

where $\delta(\theta)$ is the Dirac delta function, then (3.5) is satisfied. For $\left(\varphi_{1}, \varphi_{2}, \varphi_{3}, \varphi_{4}\right) \in\left(C^{1}[-\tau, 0]\right.$, $\left.R^{4}\right)$, define

$$
A(\mu) \varphi= \begin{cases}\frac{d \varphi(\theta)}{d \theta}, & -\tau \leq \theta<0, \\ \int_{-\tau}^{0} d \eta(s, \mu) \varphi(s), & \theta=0\end{cases}
$$

and

$$
R \varphi= \begin{cases}0, & -\tau \leq \theta<0 \\ f(\mu, \varphi), & \theta=0\end{cases}
$$

Then (1.4) is equivalent to the abstract differential equation

$$
\dot{u}_{t}=A(\mu) u_{t}+R(\mu) u_{t},
$$

where $u=\left(u_{1}, u_{2}, u_{3}, u_{4}\right)^{T}, u_{t}(\theta)=u(t+\theta), \theta \in[-\tau, 0]$.

For $\psi \in C\left([-\tau, 0],\left(R^{4}\right)^{*}\right)$, define

$$
A^{*} \psi(s)= \begin{cases}-\frac{d \psi(s)}{d s}, & s \in(0, \tau], \\ \int_{-\tau}^{0} d \eta^{T}(t, 0) \psi(-t), & s=0 .\end{cases}
$$

For $\phi \in C\left([-\tau, 0], R^{4}\right)$ and $\psi \in C\left([0, \tau],\left(R^{4}\right)^{*}\right)$, define the bilinear form

$$
\langle\psi, \phi\rangle=\bar{\psi}(0) \phi(0)-\int_{-\tau}^{0} \int_{\xi=0}^{\theta} \psi^{T}(\xi-\theta) d \eta(\theta) \phi(\xi) d \xi,
$$

where $\eta(\theta)=\eta(\theta, 0)$. We have the following result on the relation between the operators $A=A(0)$ and $A^{*}$.

Lemma 3.1 $A=A(0)$ and $A^{*}$ are adjoint operators.

Proof Let $\phi \in C^{1}\left([-\tau, 0], R^{4}\right)$ and $\psi \in C^{1}\left([0, \tau], R^{4}\right)^{*}$. It follows from (3.11) and the definitions of $A=A(0)$ and $A^{*}$ that

$$
\begin{aligned}
\langle\psi(s), A(0) \phi(\theta)\rangle & =\bar{\psi}(0) A(0) \phi(0)-\int_{-\tau_{k}^{(j)}}^{0} \int_{\xi=0}^{\theta} \bar{\psi}(\xi-\theta) d \eta(\theta) A(0) \phi(\xi) d \xi \\
& =\bar{\psi}(0) \int_{-\tau_{k}^{(j)}}^{0} d \eta(\theta) \phi(\theta)-\int_{-\tau_{k}^{(j)}}^{0} \int_{\xi=0}^{\theta} \bar{\psi}(\xi-\theta) d \eta(\theta) A(0) \phi(\xi) d \xi
\end{aligned}
$$




$$
\begin{aligned}
= & \bar{\psi}(0) \int_{-\tau_{k}^{(j)}}^{0} d \eta(\theta) \phi(\theta)-\int_{-\tau_{k}^{(j)}}^{0}[\bar{\psi}(\xi-\theta) d \eta(\theta) \phi(\xi)]_{\xi=0}^{\theta} \\
& +\int_{-\tau_{k}^{(j)}}^{0} \int_{\xi=0}^{\theta} \frac{d \bar{\psi}(\xi-\theta)}{d \xi} d \eta(\theta) \phi(\xi) d \xi \\
= & \int_{-\tau_{k}^{(j)}}^{0} \bar{\psi}(-\theta) d \eta(\theta) \phi(0)-\int_{-\tau_{k}^{(j)}}^{0} \int_{\xi=0}^{\theta}\left[-\frac{d \bar{\psi}(\xi-\theta)}{d \xi}\right] d \eta(\theta) \phi(\xi) d \xi \\
= & A * \bar{\psi}(0) \phi(0)-\int_{-\tau_{k}^{(j)}}^{0} \int_{\xi=0}^{\theta} A^{*} \bar{\psi}(\xi-\theta) d \eta(\theta) \phi(\xi) d \xi \\
= & \left\langle A^{*} \psi(s), \phi(\theta)\right\rangle .
\end{aligned}
$$

This shows that $A=A(0)$ and $A^{*}$ are adjoint operators and the proof is complete.

By the discussions in Section 2, we know that $\pm i \omega_{k}$ are eigenvalues of $A(0)$, and they are also eigenvalues of $A^{*}$ corresponding to $i \omega_{k}$ and $-i \omega_{k}$, respectively. We have the following result.

Lemma 3.2 The vector

$$
q(\theta)=\left(1, r_{1}, r_{2}, r_{3}\right)^{T} e^{i \omega_{k} \theta}, \quad \theta \in[-\tau, 0]
$$

is the eigenvector of $A(0)$ corresponding to the eigenvalue $i \omega_{k}$, and

$$
q^{*}(s)=D\left(1, r_{1}^{*}, r_{2}^{*}, r_{3}^{*}\right) e^{i \omega_{k} s}, \quad s \in[0, \tau],
$$

is the eigenvector of $A^{*}$ corresponding to the eigenvalue $-i \omega_{k}$, moreover, $\left\langle q^{*}(s), q(\theta)\right\rangle=1$, where

$$
D=\frac{1}{C}
$$

where

$$
C=1+\sum_{i=1}^{3} \bar{r}_{i} r_{i}^{*}+\left(1+\bar{r}_{1}+\bar{r}_{2}+\bar{r}_{3}\right) d_{1} r_{3}^{*} e^{i \omega_{k} \tau_{k}^{(j)}} .
$$

Proof Let $q(\theta)$ be the eigenvector of $A(0)$ corresponding to the eigenvalue $i \omega_{k}$ and $q^{*}(s)$ be the eigenvector of $A^{*}$ corresponding to the eigenvalue $-i \omega_{k}$, namely, $A(0) q(\theta)=i \omega_{k} q(\theta)$ and $A^{*} q(s)=-i \omega_{k} q^{*}(s)$. From the definitions of $A(0)$ and $A^{*}$, we have $A(0) q(\theta)=d q(\theta) / d \theta$ and $A^{*} q(s)=-d q^{*}(s) / d s$. Thus, $q(\theta)=q(0) e^{i \omega_{k} \theta}$ and $q^{*}(s)=q(0) e^{i \omega_{0} s}$. In addition,

$$
\begin{aligned}
\int_{-\tau_{k}^{(j)}}^{0} d \eta(\theta) q(\theta) & =\left(\begin{array}{cccc}
a_{1} & 0 & 0 & a_{2} \\
0 & b_{1} & 0 & b_{2} \\
0 & 0 & c_{1} & c_{2} \\
0 & 0 & 0 & 0
\end{array}\right) q(0)+\left(\begin{array}{cccc}
0 & 0 & 0 & 0 \\
0 & 0 & 0 & 0 \\
0 & 0 & 0 & 0 \\
d_{1} & d_{1} & d_{1} & 0
\end{array}\right) q\left(-\tau_{k}^{(j)}\right) \\
& =A(0) q(0)=i \omega_{k} q(0) .
\end{aligned}
$$


That is,

$$
\left(\begin{array}{c}
a_{1}+a_{2} r_{3} \\
b_{1} r_{1}+b_{2} r_{3} \\
c_{1} r_{2}+c_{2} r_{3} \\
d_{1}\left(1+r_{1}+r_{2}\right) e^{-i \omega_{k} \tau_{k}^{(j)}}
\end{array}\right)=\left(\begin{array}{c}
i \omega_{k} \\
i \omega_{k} r_{1} \\
i \omega_{k} r_{2} \\
i \omega_{k} r_{3}
\end{array}\right)
$$

Therefore, we can easily obtain

$$
r_{1}=\frac{b_{2}\left(a_{1}-i \omega_{k}\right)}{a_{2}\left(b_{1}-i \omega_{k}\right)}, \quad r_{2}=\frac{c_{2}\left(a_{1}-i \omega_{k}\right)}{a_{2}\left(c_{1}-i \omega_{k}\right)}, \quad r_{3}=\frac{i \omega_{k}-a_{1}}{a_{2}}
$$

On the other hand,

$$
\begin{aligned}
\int_{-\tau_{k}^{(j)}}^{0} q^{*}(-t) d \eta(t) & =\left(\begin{array}{cccc}
a_{1} & 0 & 0 & 0 \\
0 & b_{1} & 0 & 0 \\
0 & 0 & c_{1} & 0 \\
a_{2} & b_{2} & c_{2} & 0
\end{array}\right) q(0)+\left(\begin{array}{cccc}
0 & 0 & 0 & d_{1} \\
0 & 0 & 0 & d_{1} \\
0 & 0 & 0 & d_{1} \\
0 & 0 & 0 & 0
\end{array}\right) q^{*}\left(-\tau_{k}^{(j)}\right) \\
& =A^{*} q^{*}(0)=-i \omega_{0} q^{*}(0) .
\end{aligned}
$$

Namely,

$$
\left(\begin{array}{c}
a_{1}+d_{1} r_{3}^{*} e^{-i \omega_{k} \tau_{k}^{(j)}} \\
b_{1} r_{1}^{*}+d_{1} r_{3}^{*} e^{-i \omega_{k} \tau_{k}^{(j)}} \\
c_{1} r_{2}^{*}+d_{1} r_{3}^{*} e^{-i \omega_{k} \tau_{k}^{(j)}} \\
a_{2}+b_{2} r_{1}^{*}+c_{2} r_{3}^{*}
\end{array}\right)=\left(\begin{array}{c}
-i \omega_{k} \\
-i \omega_{k} r_{1}^{*} \\
-i \omega_{k} r_{2}^{*} \\
-i \omega_{k} r_{3}^{*}
\end{array}\right)
$$

Therefore, we can easily obtain

$$
r_{1}^{*}=\frac{a_{1}+i \omega_{k}}{b_{1}+i \omega_{k}}, \quad r_{2}^{*}=\frac{a_{1}+i \omega_{k}}{c_{1}+i \omega_{k}}, \quad r_{3}^{*}=-\frac{a_{1}+i \omega_{k}}{d_{1} e^{-i \omega_{k} \tau_{k}^{(j)}}}
$$

In the sequel, we shall verify that $\left\langle q^{*}(s), q(\theta)\right\rangle=1$. In fact, from (3.11), we have

$$
\begin{aligned}
\left\langle q^{*}(s), q(\theta)\right\rangle= & \bar{D}\left(1, \bar{r}_{1}^{*}, \bar{r}_{2}^{*}, \bar{r}_{3}^{*}\right)\left(1, r_{1}, r_{2}, r_{3}\right)^{T} \\
& -\int_{-\tau_{k}^{(j)}}^{0} \int_{\xi=0}^{\theta} \bar{D}\left(1, \bar{r}_{1}^{*}, \bar{r}_{2}^{*}, \bar{r}_{3}^{*}\right) e^{-i \omega_{k}(\xi-\theta)} d \eta(\theta)\left(1, r_{1}, r_{2}, r_{3}\right)^{T} e^{i \omega_{k} \xi} d \xi \\
= & \bar{D}\left[1+\sum_{i=1}^{3} r_{i} \bar{r}_{i}^{*}-\int_{-\tau_{k}^{(j)}}^{0}\left(1, \bar{r}_{1}^{*}, \bar{r}_{2}^{*}, \bar{r}_{3}^{*}\right) \theta e^{i \omega_{k} \theta} d \eta(\theta)\left(1, r_{1}, r_{2}, r_{3}\right)^{T}\right] \\
= & \bar{D}\left\{1+\sum_{i=1}^{3} r_{i} \bar{r}_{i}^{*}+\left(1, \bar{r}_{1}^{*}, \bar{r}_{2}^{*}, \bar{r}_{3}^{*}\right) B_{1} e^{\left.-i \omega_{k} \tau_{k}^{(j)}\left(1, r_{1}, r_{2}, r_{3}\right)^{T}\right\}}\right. \\
= & \bar{D}\left[1+\sum_{i=1}^{3} r_{i} \bar{r}_{i}^{*}+\left(1+r_{1}+r_{2}+r_{3}\right) d_{1} \bar{r}_{3}^{*} e^{-i \omega_{k} \tau_{k}^{(j)}}\right]=1 .
\end{aligned}
$$


Next, we use the same notations as those in Hassard et al. [15], and we first compute the coordinates to describe the center manifold $C_{0}$ at $\mu=0$. Let $y_{t}$ be the solution of equation (1.4) when $\mu=0$.

Define

$$
z(t)=\left\langle q^{*}, y_{t}\right\rangle, \quad W(t, \theta)=y_{t}(\theta)-2 \operatorname{Re}\{z(t) q(\theta)\}
$$

on the center manifold $C_{0}$, and we have

$$
W(t, \theta)=W(z(t), \bar{z}(t), \theta)
$$

where

$$
W(z(t), \bar{z}(t), \theta)=W(z, \bar{z})=W_{20} \frac{z^{2}}{2}+W_{11} z \bar{z}+W_{02} \frac{\bar{z}^{2}}{2}+\cdots
$$

and $z$ and $\bar{z}$ are local coordinates for center manifold $C_{0}$ in the direction of $q^{*}$ and $\bar{q}^{*}$. Noting that $W$ is also real if $y_{t}$ is real, we consider only real solutions. For solutions $y_{t} \in C_{0}$ of (1.4),

$$
\begin{aligned}
\dot{z}(t)= & \left\langle q^{*}(s), \dot{x}_{t}\right\rangle=\left\langle q^{*}(s), A(0) u_{t}+R(0) u_{t}\right\rangle \\
= & \left\langle q^{*}(s), A(0) y_{t}\right\rangle+\left\langle q^{*}(s), R(0) y_{t}\right\rangle \\
= & \left\langle A^{*} q^{*}(s), y_{t}\right\rangle+\bar{q}^{*}(0) R(0) y_{t} \\
& -\int_{-\tau_{k}^{(j)}}^{0} \int_{\xi=0}^{\theta} \bar{q}^{*}(\xi-\theta) d \eta(\theta) A(0) R(0) y_{t}(\xi) d \xi \\
= & \left\langle i \omega_{k} q^{*}(s), y_{t}\right\rangle+\bar{q}^{*}(0) f\left(0, y_{t}(\theta)\right) \\
\stackrel{\text { def }}{=} & i \omega_{k} z(t)+\bar{q}^{*}(0) f_{0}(z(t), \bar{z}(t)) .
\end{aligned}
$$

That is,

$$
\dot{z}(t)=i \omega_{k} z+g(z, \bar{z}),
$$

where

$$
g(z, \bar{z})=g_{20} \frac{z^{2}}{2}+g_{11} z \bar{z}+g_{02} \frac{\bar{z}^{2}}{2}+g_{21} \frac{z^{2} \bar{z}}{2}+\cdots .
$$

Hence, we have

$$
\begin{aligned}
g(z, \bar{z}) & =\bar{q}^{*}(0) f_{0}(z, \bar{z})=f\left(0, y_{t}\right) \\
& =\bar{D}\left(1, \bar{r}_{1}^{*}, \bar{r}_{2}^{*}, \bar{r}_{3}^{*}\right)\left(f_{1}\left(0, y_{t}\right), f_{2}\left(0, y_{t}\right), f_{3}\left(0, y_{t}\right), f_{4}\left(0, y_{t}\right)\right)^{T}
\end{aligned}
$$

where

$$
\begin{aligned}
f_{1}\left(0, y_{t}\right)= & a_{3} y_{1 t}^{2}(0)+a_{4} y_{1 t}(0) y_{1 t}\left(-\tau_{k}^{(j)}\right)+a_{5} y_{1 t}(0) y_{4 t}(0)+a_{6} y_{1 t}\left(-\tau_{k}^{(j)}\right) y_{4 t}(0) \\
& +a_{7} y_{1 t}^{3}(0)+a_{8} y_{1 t}^{2}(0) y_{1 t}\left(-\tau_{k}^{(j)}\right)+a_{9} y_{1 t}^{2}(0) y_{4 t}(0)
\end{aligned}
$$




$$
\begin{aligned}
& +a_{10} y_{1 t}(0) y_{1 t}\left(-\tau_{k}^{(j)}\right) y_{4 t}(0)+\text { h.o.t., } \\
f_{2}\left(0, y_{t}\right)= & b_{3} y_{2 t}^{2}(0)+b_{4} y_{2 t}(0) y_{2 t}\left(-\tau_{k}^{(j)}\right)+b_{5} y_{2 t}(0) y_{4 t}(0)+b_{6} y_{2 t}\left(-\tau_{k}^{(j)}\right) y_{4 t}(0) \\
& +b_{7} y_{2 t}^{3}(0)+b_{8} y_{2 t}^{2}(0) y_{2 t}\left(-\tau_{k}^{(j)}\right)+b_{9} y_{2 t}^{2}(0) y_{4 t}(0) \\
& +b_{10} y_{2 t}(0) y_{2 t}\left(-\tau_{k}^{(j)}\right) y_{4 t}(0)+\text { h.o.t., } \\
f_{3}\left(0, y_{t}\right)= & c_{3} y_{3 t}^{2}(0)+c_{4} y_{3 t}(0) y_{3 t}\left(-\tau_{k}^{(j)}\right)+c_{5} y_{3 t}(0) y_{4 t}(0)+c_{6} y_{3 t}\left(-\tau_{k}^{(j)}\right) y_{4 t}(0) \\
& +c_{7} y_{3 t}^{3}(0)+c_{8} y_{3 t}^{2}(0) y_{3 t}\left(-\tau_{k}^{(j)}\right)+c_{9} y_{3 t}^{2}(0) y_{4 t}(0) \\
& +c_{10} y_{3 t}(0) y_{3 t}\left(-\tau_{k}^{(j)}\right) y_{4 t}(0)+\text { h.o.t., } \\
f_{4}\left(0, y_{t}\right)= & d_{1} y_{1 t}\left(-\tau_{k}^{(j)}\right) y_{4 t}(0)+d_{1} y_{2 t}\left(-\tau_{k}^{(j)}\right) y_{4 t}(0)+d_{1} y_{3 t}\left(-\tau_{k}^{(j)}\right) y_{4 t}(0) .
\end{aligned}
$$

Noticing that

$$
y_{t}(\theta)=\left(y_{1 t}(\theta), y_{2 t}(\theta), y_{3 t}(\theta), y_{4 t}(\theta)\right)^{T}=W(t, \theta)+z q(\theta)+\bar{z} \bar{q}
$$

and

$$
q(\theta)=\left(1, r_{1}, r_{2}, r_{3}\right)^{T} e^{i \omega_{k} \theta},
$$

we have

$$
\begin{aligned}
& y_{1 t}(0)=z+\bar{z}+W_{20}^{(1)}(0) \frac{z^{2}}{2}+W_{11}^{(1)}(0) z \bar{z}+W_{02}^{(1)}(0) \frac{\bar{z}^{2}}{2}+\cdots, \\
& y_{2 t}(0)=r_{1} z+\bar{r}_{1} \bar{z}+W_{20}^{(2)}(0) \frac{z^{2}}{2}+W_{11}^{(2)}(0) z \bar{z}+W_{02}^{(2)}(0) \frac{\bar{z}^{2}}{2}+\cdots, \\
& y_{3 t}(0)=r_{2} z+\bar{r}_{2} \bar{z}+W_{20}^{(3)}(0) \frac{z^{2}}{2}+W_{11}^{(3)}(0) z \bar{z}+W_{02}^{(3)}(0) \frac{\bar{z}^{2}}{2}+\cdots, \\
& y_{4 t}(0)=r_{3} z+\bar{r}_{3} \bar{z}+W_{20}^{(4)}(0) \frac{z^{2}}{2}+W_{11}^{(4)}(0) z \bar{z}+W_{02}^{(4)}(0) \frac{\bar{z}^{2}}{2}+\cdots, \\
& y_{1 t}\left(-\tau_{k}^{(j)}\right)=e^{-i \omega_{k} \tau_{k}^{(j)}} z+e^{i \omega_{k} \tau_{k}^{(j)}} \bar{z}+W_{20}^{(1)}\left(-\tau_{k}^{(j)}\right) \frac{z^{2}}{2} \\
& +W_{11}^{(1)}\left(-\tau_{k}^{(j)}\right) z \bar{z}+W_{02}^{(1)}\left(-\tau_{k}^{(j)}\right) \frac{\bar{z}^{2}}{2}+\cdots, \\
& y_{2 t}\left(-\tau_{k}^{(j)}\right)=r_{1} e^{-i \omega_{k} \tau_{k}^{(j)}} z+\bar{r}_{1} e^{i \omega_{k} \tau_{k}^{(j)}} \bar{z}+W_{20}^{(2)}\left(-\tau_{k}^{(j)}\right) \frac{z^{2}}{2} \\
& +W_{11}^{(2)}\left(-\tau_{k}^{(j)}\right) z \bar{z}+W_{02}^{(2)}\left(-\tau_{k}^{(j)}\right) \frac{\bar{z}^{2}}{2}+\cdots, \\
& y_{3 t}\left(-\tau_{k}^{(j)}\right)=r_{2} e^{-i \omega_{k} \tau_{k}^{(j)}} z+\bar{r}_{2} e^{i \omega_{k} \tau_{k}^{(j)}} \bar{z}+W_{20}^{(3)}\left(-\tau_{k}^{(j)}\right) \frac{z^{2}}{2} \\
& +W_{11}^{(3)}\left(-\tau_{k}^{(j)}\right) z \bar{z}+W_{02}^{(3)}\left(-\tau_{k}^{(j)}\right) \frac{\bar{z}^{2}}{2}+\cdots, \\
& y_{4 t}\left(-\tau_{k}^{(j)}\right)=r_{3} e^{-i \omega_{k} \tau_{k}^{(j)}} z+\bar{r}_{3} e^{i \omega_{k} \tau_{k}^{(j)}} \bar{z}+W_{20}^{(4)}\left(-\tau_{k}^{(j)}\right) \frac{z^{2}}{2} \\
& +W_{11}^{(4)}\left(-\tau_{k}^{(j)}\right) z \bar{z}+W_{02}^{(4)}\left(-\tau_{k}^{(j)}\right) \frac{\bar{z}^{2}}{2}+\cdots .
\end{aligned}
$$


Xu and Li Advances in Difference Equations （2016） 2016:40

Page 14 of 24

From (3.22) and (3.23), we have

$$
\begin{aligned}
& g(z, \bar{z})=\bar{q}^{*}(0) f_{0}(z, \bar{z}) \\
& =\bar{D}\left[f_{1}\left(0, y_{t}\right)+\bar{r}_{1}^{*} f_{2}\left(0, x_{t}\right)+\bar{r}_{2}^{*} f_{3}\left(0, y_{t}\right)+\bar{r}_{3}^{*} f_{4}\left(0, y_{t}\right)\right] \\
& =\bar{D}\left[a_{3}+a_{4} e^{-i \omega_{k} \tau_{k}^{(j)}}+a_{5} r_{3}+a_{6} r_{3} e^{-i \omega_{k} \tau_{k}^{(j)}}\right. \\
& +\bar{r}_{1}^{*}\left(b_{3} r_{1}^{2}+b_{4} r_{1}^{2} e^{-i \omega_{k} \tau_{k}^{(j)}}+b_{5} r_{1} r_{3}+b_{6} r_{1} r_{3} e^{-i \omega_{k} \tau_{k}^{(j)}}\right) \\
& +\bar{r}_{2}^{*}\left(c_{3} r_{2}^{2}+c_{4} r_{2}^{2} e^{-i \omega_{k} \tau_{k}^{(j)}}+c_{5} r_{2} r_{3}+c_{6} r_{2} r_{3} e^{-i \omega_{k} \tau_{k}^{(j)}}\right) \\
& \left.+\bar{r}_{3}^{*}\left(d_{1} r_{3} e^{-i \omega_{k} \tau_{k}^{(j)}}+d_{1} r_{1} r_{3} e^{-i \omega_{k} \tau_{k}^{(j)}}+d_{1} r_{2} r_{3} e^{-i \omega_{k} \tau_{k}^{(j)}}\right)\right] z^{2} \\
& +\bar{D}\left[2 a_{3}+2 a_{4} \operatorname{Re}\left\{r_{3} e^{i \omega_{k} \tau_{k}^{(j)}}\right\}+2 a_{5} \operatorname{Re}\left\{r_{3}\right\}+2 a_{6} \operatorname{Re}\left\{r_{3} e^{i \omega_{k} \tau_{k}^{(j)}}\right\}\right. \\
& +r_{1}^{*}\left(2 b_{3}\left|r_{1}\right|^{2}+2 b_{4} \operatorname{Re}\left\{r_{1} \bar{r}_{1} e^{-i \omega_{k} \tau_{k}^{(j)}}\right\}+2 b_{5} \operatorname{Re}\left\{r_{1} \bar{r}_{3}\right\}+2 b_{6} \operatorname{Re}\left\{r_{1} \bar{r}_{3} e^{-i \omega_{k} \tau_{k}^{(j)}}\right\}\right) \\
& +r_{2}^{*}\left(2 c_{3}\left|r_{2}\right|^{2}+2 c_{4} \operatorname{Re}\left\{\left|r_{2}\right|^{2} e^{i \omega_{k} \tau_{k}^{(j)}}\right\}+2 c_{5} \operatorname{Re}\left\{r_{2} \bar{r}_{3}\right\}+2 c_{6} \operatorname{Re}\left\{r_{2} \bar{r}_{3} e^{-i \omega_{k} \tau_{k}^{(j)}}\right\}\right) \\
& \left.+r_{3}^{*}\left(2 d_{1} \operatorname{Re}\left\{r_{3} e^{i \omega_{k} \tau_{k}^{(j)}}\right\}+2 d_{1} \operatorname{Re}\left\{r_{1} e^{-i \omega_{k} \tau_{k}^{(j)}}\right\}+2 d_{1} \operatorname{Re}\left\{r_{2} e^{-i \omega_{k} \tau_{k}^{(j)}}\right\}\right)\right] z \bar{z} \\
& +\bar{D}\left[a_{3}+a_{4} e^{i \omega_{k} \tau_{k}^{(j)}}+a_{5} \bar{r}_{3}+a_{6} \bar{r}_{3} e^{i \omega_{k} \tau_{k}^{(j)}}\right. \\
& +r_{1}^{*}\left(b_{3} \bar{r}_{1}^{2}+b_{4} \bar{r}_{1}^{2} e^{i \omega_{k} \tau_{k}^{(j)}}+b_{5} \bar{r}_{1} \bar{r}_{3}+b_{6} \bar{r}_{1} \bar{r}_{3} e^{i \omega_{k} \tau_{k}^{(j)}}\right) \\
& +r_{2}^{*}\left(c_{3} \bar{r}_{2}^{2}+c_{4} \bar{r}_{2}^{2} e^{i \omega_{k} \tau_{k}^{(j)}}+c_{5} \bar{r}_{2} r_{3}+c_{6} \bar{r}_{2} \bar{r}_{3} e^{i \omega_{k} \tau_{k}^{(j)}}\right) \\
& \left.+r_{3}\left(d_{1} \bar{r}_{3} e^{i \omega_{k} \tau_{k}^{(j)}}+d_{1} \bar{r}_{1} \bar{r}_{3} e^{i \omega_{k} \tau_{k}^{(j)}}+d_{1} \bar{r}_{2} \bar{r}_{3} e^{i \omega_{k} \tau_{k}^{(j)}}\right)\right] \bar{z}^{2} \\
& +\bar{D}\left\{r _ { 1 } ^ { * } \left[b_{3}\left(2 r_{1} W_{11}^{(2)}(0)+W_{201}^{(2)}(0) \bar{r}_{1}\right)\right.\right. \\
& +b_{4}\left(r_{1} W_{11}^{(2)}\left(-\tau_{k}^{(j)}\right)+\frac{1}{2} \bar{r}_{1} W_{20}^{(2)}\left(-\tau_{k}^{(j)}\right)\right. \\
& \left.+\frac{1}{2} \bar{r}_{1} W_{20}^{(2)}(0) e^{-i \omega_{k} \tau_{k}^{(j)}}+r_{1} W_{11}^{(2)}(0) e^{-i \omega_{k} \tau_{k}^{(j)}}\right)+b_{5}\left(r_{1} W_{11}^{(4)}(0)+\frac{1}{2} \bar{r}_{1} W_{20}^{(4)}(0)\right. \\
& \left.+\frac{1}{2} \bar{r}_{3} W_{20}^{(2)}(0)+r_{3} W_{11}^{(2)}(0)\right)+b_{6}\left(r_{1} W_{11}^{(4)}(0)+\frac{1}{2} \bar{r}_{1} W_{20}^{(4)}(0) e^{i \omega_{k} \tau_{k}^{(j)}}\right. \\
& \left.+\frac{1}{2} \bar{r}_{3} W_{20}^{(2)}(-\tau) e^{i \omega_{k} \tau_{k}^{(j)}}+r_{3} W_{11}^{(2)}\left(-\tau_{k}^{(j)}\right)\right) \\
& +3 b_{7} r_{1}^{2} \bar{r}_{1}+b_{8}\left(r_{1}^{2} \bar{r}_{1} e^{i \omega_{k} \tau_{k}^{(j)}}+2 r_{1}^{2} \bar{r}_{1} e^{-i \omega_{k} \tau_{k}^{(j)}}\right) \\
& \left.+b_{9}\left(r_{1}^{2} \bar{r}_{3}+2\left|r_{1}\right|^{2} r_{3}\right)+b_{10}\left(r_{1}^{2} e^{-i \omega_{k} \tau_{k}^{(j)}}+\left|r_{1}\right|^{2} r_{3} e^{i \omega_{k} \tau_{k}^{(j)}}+\left|r_{1}\right|^{2} r_{3} e^{-i \omega_{k} \tau_{k}^{(j)}}\right)\right] \\
& +r_{2}^{*}\left[3 c_{3} r_{2}^{2} \bar{r}_{2}+c_{4}\left(r_{2} W_{11}^{(3)}\left(-\tau_{k}^{(j)}\right)+\frac{1}{2} \bar{r}_{2} W_{20}^{(3)}\left(-\tau_{k}^{(j)}\right)\right.\right. \\
& \left.+\frac{1}{2} \bar{r}_{2} W_{20}^{(3)}\left(-\tau_{k}^{(j)}\right) e^{i \omega_{k} \tau_{k}^{(j)}}+r_{2} W_{11}^{(3)}(0) e^{-i \omega_{k} \tau_{k}^{(j)}}\right) \\
& +c_{5}\left(r_{2} W_{11}^{(4)}(0)+\frac{1}{2} \bar{r}_{2} W_{20}^{(4)}(0)+\frac{1}{2} \bar{r}_{3} W_{20}^{(3)}(0)+r_{3} W_{11}^{(3)}(0)\right) \\
& +c_{6}\left(r_{2} W_{11}^{(4)}(0) e^{-i \omega_{k} \tau_{k}^{(j)}}+\frac{1}{2} \bar{r}_{2} W_{20}^{(4)}(0) e^{i \omega_{k} \tau_{k}^{(j)}}\right.
\end{aligned}
$$


Xu and Li Advances in Difference Equations （2016） 2016:40

Page 15 of 24

$$
\begin{aligned}
& \left.+\frac{1}{2} \bar{r}_{3} W_{20}^{(3)}\left(-\tau_{k}^{(j)}\right)+r_{3} W_{11}^{(3)}\left(-\tau_{k}^{(j)}\right)\right) \\
& +3 c_{7} r_{2}^{2} \bar{r}_{2}+c_{8}\left(r_{2}^{2} \bar{r}_{2} e^{i \omega_{k} \tau_{k}^{(j)}}+2 r_{2}^{2} \bar{r}_{2} e^{-i \omega_{k} \tau_{k}^{(j)}}\right) \\
& +c_{9}\left(r_{2}^{2} \bar{r}_{3}+2\left|r_{2}\right|^{2} r_{3}\right)+c_{10}\left(r_{2}^{2} \bar{r}_{3} e^{-i \omega_{k} \tau_{k}^{(j)}}\right. \\
& \left.+\left|r_{2}\right|^{2} r_{3} e^{-i \omega_{k} \tau_{k}^{(j)}}+\left|r_{2}\right|^{2} r_{3} e^{\left.i \omega_{k} \tau_{k}^{(j)}\right)}\right]+\bar{r}_{3}^{*} d_{1}\left[W_{11}^{(4)}(0)+\frac{1}{2} W_{20}^{(4)}(0)+\frac{1}{2} \bar{r}_{3} W_{20}^{(1)}(0)\right. \\
& +r_{3} W_{11}^{(1)}(0)+r_{1} W_{11}^{(4)}(0) e^{-i \omega_{k} \tau_{k}^{(j)}}+\frac{1}{2} \bar{r}_{1} W_{20}^{(4)}(0) e^{i \omega_{k} \tau_{k}^{(j)}} \\
& +\frac{1}{2} \bar{r}_{3} W_{20}^{(4)}\left(-\tau_{k}^{(j)}\right)+r_{3} W_{11}^{(2)}\left(-\tau_{k}^{(j)}\right)+r_{2} W_{11}^{(4)}(0) e^{-i \omega_{k} \tau_{k}^{(j)}} \\
& \left.\left.+\frac{1}{2} \bar{r}_{2} W_{20}^{(4)}(0) e^{i \omega_{k} \tau_{k}^{(j)}}+\frac{1}{2} \bar{r}_{3} W_{20}^{(3)}\left(-\tau_{k}^{(j)}\right) e^{i \omega_{k} \tau_{k}^{(j)}}+r_{3} W_{11}^{(3)}\left(-\tau_{k}^{(j)}\right)\right] z^{2} \bar{z}+\cdots\right\}
\end{aligned}
$$

and we obtain

$$
\begin{aligned}
& g_{20}=2 \bar{D}\left[a_{3}+a_{4} e^{-i \omega_{k} \tau_{k}^{(j)}}+a_{5} r_{3}+a_{6} r_{3} e^{-i \omega_{k} \tau_{k}^{(j)}}\right. \\
& +\bar{r}_{1}^{*}\left(b_{3} r_{1}^{2}+b_{4} r_{1}^{2} e^{-i \omega_{k} \tau_{k}^{(j)}}+b_{5} r_{1} r_{3}+b_{6} r_{1} r_{3} e^{-i \omega_{k} \tau_{k}^{(j)}}\right) \\
& +\bar{r}_{2}^{*}\left(c_{3} r_{2}^{2}+c_{4} r_{2}^{2} e^{-i \omega_{k} \tau_{k}^{(j)}}+c_{5} r_{2} r_{3}+c_{6} r_{2} r_{3} e^{-i \omega_{k} \tau_{k}^{(j)}}\right) \\
& \left.+\bar{r}_{3}^{*}\left(d_{1} r_{3} e^{-i \omega_{k} \tau_{k}^{(j)}}+d_{1} r_{1} r_{3} e^{-i \omega_{k} \tau_{k}^{(j)}}+d_{1} r_{2} r_{3} e^{-i \omega_{k} \tau_{k}^{(j)}}\right)\right], \\
& g_{11}=\bar{D}\left[2 a_{3}+2 a_{4} \operatorname{Re}\left\{r_{3} e^{i \omega_{k} \tau_{k}^{(j)}}\right\}+2 a_{5} \operatorname{Re}\left\{r_{3}\right\}+2 a_{6} \operatorname{Re}\left\{r_{3} e^{i \omega_{k} \tau_{k}^{(j)}}\right\}\right. \\
& +r_{1}^{*}\left(2 b_{3}\left|r_{1}\right|^{2}+2 b_{4} \operatorname{Re}\left\{r_{1} \bar{r}_{1} e^{-i \omega_{k} \tau_{k}^{(j)}}\right\}+2 b_{5} \operatorname{Re}\left\{r_{1} \bar{r}_{3}\right\}+2 b_{6} \operatorname{Re}\left\{r_{1} \bar{r}_{3} e^{-i \omega_{k} \tau_{k}^{(j)}}\right\}\right) \\
& +r_{2}^{*}\left(2 c_{3}\left|r_{2}\right|^{2}+2 c_{4} \operatorname{Re}\left\{\left|r_{2}\right|^{2} e^{i \omega_{0} \tau_{k}^{(j)}}\right\}+2 c_{5} \operatorname{Re}\left\{r_{2} \bar{r}_{3}\right\}+2 c_{6} \operatorname{Re}\left\{r_{2} \bar{r}_{3} e^{-i \omega_{k} \tau_{k}^{(j)}}\right\}\right) \\
& \left.+r_{3}^{*}\left(2 d_{1} \operatorname{Re}\left\{r_{3} e^{i \omega_{k} \tau_{k}^{(j)}}\right\}+2 d_{1} \operatorname{Re}\left\{r_{1} e^{-i \omega_{k} \tau_{k}^{(j)}}\right\}+2 d_{1} \operatorname{Re}\left\{r_{2} e^{-i \omega_{k} \tau_{k}^{(j)}}\right\}\right)\right], \\
& g_{02}=2 \bar{D}\left[a_{3}+a_{4} e^{i \omega_{k} \tau_{k}^{(j)}}+a_{5} \bar{r}_{3}+a_{6} \bar{r}_{3} e^{i \omega_{k} \tau_{k}^{(j)}}\right. \\
& +r_{1}^{*}\left(b_{3} \bar{r}_{1}^{2}+b_{4} \bar{r}_{1}^{2} e^{i \omega_{k} \tau_{k}^{(j)}}+b_{5} \bar{r}_{1} \bar{r}_{3}+b_{6} \bar{r}_{1} \bar{r}_{3} e^{i \omega_{k} \tau_{k}^{(j)}}\right) \\
& +r_{2}^{*}\left(c_{3} \bar{r}_{2}^{2}+c_{4} \bar{r}_{2}^{2} e^{i \omega_{k} \tau_{k}^{(j)}}+c_{5} \bar{r}_{2} r_{3}+c_{6} \bar{r}_{2} \bar{r}_{3} e^{i \omega_{k} \tau_{k}^{(j)}}\right) \\
& \left.+r_{3}\left(d_{1} \bar{r}_{3} e^{i \omega_{k} \tau_{k}^{(j)}}+d_{1} \bar{r}_{1} \bar{r}_{3} e^{i \omega_{k} \tau_{k}^{(j)}}+d_{1} \bar{r}_{2} \bar{r}_{3} e^{i \omega_{k} \tau_{k}^{(j)}}\right)\right], \\
& g_{21}=2 \bar{D}\left\{r _ { 1 } ^ { * } \left[b_{3}\left(2 r_{1} W_{11}^{(2)}(0)+W_{201}^{(2)}(0) \bar{r}_{1}\right)+b_{4}\left(r_{1} W_{11}^{(2)}\left(-\tau_{k}^{(j)}\right)+\frac{1}{2} \bar{r}_{1} W_{20}^{(2)}\left(-\tau_{k}^{(j)}\right)\right.\right.\right. \\
& \left.+\frac{1}{2} \bar{r}_{1} W_{20}^{(2)}(0) e^{-i \omega_{k} \tau_{k}^{(j)}}+r_{1} W_{11}^{(2)}(0) e^{-i \omega_{k} \tau_{k}^{(j)}}\right)+b_{5}\left(r_{1} W_{11}^{(4)}(0)+\frac{1}{2} \bar{r}_{1} W_{20}^{(4)}(0)\right. \\
& \left.+\frac{1}{2} \bar{r}_{3} W_{20}^{(2)}(0)+r_{3} W_{11}^{(2)}(0)\right)+b_{6}\left(r_{1} W_{11}^{(4)}(0)+\frac{1}{2} \bar{r}_{1} W_{20}^{(4)}(0) e^{i \omega_{k} \tau_{k}^{(j)}}\right. \\
& \left.+\frac{1}{2} \bar{r}_{3} W_{20}^{(2)}\left(-\tau_{k}^{(j)}\right) e^{i \omega_{k} \tau_{k}^{(j)}}+r_{3} W_{11}^{(2)}\left(-\tau_{k}^{(j)}\right)\right) \\
& +3 b_{7} r_{1}^{2} \bar{r}_{1}+b_{8}\left(r_{1}^{2} \bar{r}_{1} e^{i \omega_{k} \tau_{k}^{(j)}}+2 r_{1}^{2} \bar{r}_{1} e^{-i \omega_{k} \tau_{k}^{(j)}}\right) \\
& \left.+b_{9}\left(r_{1}^{2} \bar{r}_{3}+2\left|r_{1}\right|^{2} r_{3}\right)+b_{10}\left(r_{1}^{2} e^{-i \omega_{k} \tau_{k}^{(j)}}+\left|r_{1}\right|^{2} r_{3} e^{i \omega_{k} \tau_{k}^{(j)}}+\left|r_{1}\right|^{2} r_{3} e^{-i \omega_{k} \tau_{k}^{(j)}}\right)\right]
\end{aligned}
$$




$$
\begin{aligned}
& +r_{2}^{*}\left[3 c_{3} r_{2}^{2} \bar{r}_{2}+c_{4}\left(r_{2} W_{11}^{(3)}\left(-\tau_{k}^{(j)}\right)+\frac{1}{2} \bar{r}_{2} W_{20}^{(3)}\left(-\tau_{k}^{(j)}\right)+\frac{1}{2} \bar{r}_{2} W_{20}^{(3)}(-\tau) e^{i \omega_{k} \tau_{k}^{(j)}}\right.\right. \\
& \left.+r_{2} W_{11}^{(3)}(0) e^{-i \omega_{k} \tau_{k}^{(j)}}\right)+c_{5}\left(r_{2} W_{11}^{(4)}(0)+\frac{1}{2} \bar{r}_{2} W_{20}^{(4)}(0)+\frac{1}{2} \bar{r}_{3} W_{20}^{(3)}(0)+r_{3} W_{11}^{(3)}(0)\right) \\
& +c_{6}\left(r_{2} W_{11}^{(4)}(0) e^{-i \omega_{k} \tau_{k}^{(j)}}+\frac{1}{2} \bar{r}_{2} W_{20}^{(4)}(0) e^{i \omega_{k} \tau_{k}^{(j)}}+\frac{1}{2} \bar{r}_{3} W_{20}^{(3)}\left(-\tau_{k}^{(j)}\right)+r_{3} W_{11}^{(3)}\left(-\tau_{k}^{(j)}\right)\right) \\
& +3 c_{7} r_{2}^{2} \bar{r}_{2}+c_{8}\left(r_{2}^{2} \bar{r}_{2} e^{i \omega_{k} \tau_{k}^{(j)}}+2 r_{2}^{2} \bar{r}_{2} e^{-i \omega_{k} \tau_{k}^{(j)}}\right)+c_{9}\left(r_{2}^{2} \bar{r}_{3}+2\left|r_{2}\right|^{2} r_{3}\right)+c_{10}\left(r_{2}^{2} \bar{r}_{3} e^{-i \omega_{k} \tau_{k}^{(j)}}\right. \\
& \left.+\left|r_{2}\right|^{2} r_{3} e^{-i \omega_{k} \tau_{k}^{(j)}}+\left|r_{2}\right|^{2} r_{3} e^{\left.i \omega_{k} \tau_{k}^{(j)}\right)}\right]+\bar{r}_{3}^{*} d_{1}\left[W_{11}^{(4)}(0)+\frac{1}{2} W_{20}^{(4)}(0)+\frac{1}{2} \bar{r}_{3} W_{20}^{(1)}(0)\right. \\
& +r_{3} W_{11}^{(1)}(0)+r_{1} W_{11}^{(4)}(0) e^{-i \omega_{k} \tau_{k}^{(j)}}+\frac{1}{2} \bar{r}_{1} W_{20}^{(4)}(0) e^{i \omega_{k} \tau_{k}^{(j)}} \\
& +\frac{1}{2} \bar{r}_{3} W_{20}^{(4)}\left(-\tau_{k}^{(j)}\right)+r_{3} W_{11}^{(2)}\left(-\tau_{k}^{(j)}\right)+r_{2} W_{11}^{(4)}(0) e^{-i \omega_{k} \tau_{k}^{(j)}} \\
& \left.\left.+\frac{1}{2} \bar{r}_{2} W_{20}^{(4)}(0) e^{i \omega_{k} \tau_{k}^{(j)}}+\frac{1}{2} \bar{r}_{3} W_{20}^{(3)}\left(-\tau_{k}^{(j)}\right) e^{i \omega_{k} \tau_{k}^{(j)}}+r_{3} W_{11}^{(3)}\left(-\tau_{k}^{(j)}\right)\right]\right\}
\end{aligned}
$$

For

$$
\begin{array}{llllll}
W_{20}^{(2)}(0), & W_{11}^{(2)}(0), & W_{11}^{(2)}\left(-\tau_{k}^{(j)}\right), & W_{20}^{(2)}\left(-\tau_{k}^{(j)}\right), & W_{11}^{(4)}(0), & W_{20}^{(4)}(0), \\
W_{11}^{(4)}(0), & W_{20}^{(4)}(0), & W_{11}^{(2)}\left(-\tau_{k}^{(j)}\right), & W_{11}^{(3)}\left(-\tau_{k}^{(j)}\right), & W_{11}^{(2)}(0), & W_{20}^{(3)}(0), \\
W_{11}^{(3)}(0), & W_{11}^{(4)}(0), & W_{20}^{(3)}\left(-\tau_{k}^{(j)}\right), & W_{11}^{(3)}\left(-\tau_{k}^{(j)}\right), & W_{20}^{(3)}\left(-\tau_{k}^{(j)}\right) &
\end{array}
$$

unknown in $g_{21}$, we still need to compute them.

From (3.9), (3.23), we have

$$
\begin{aligned}
& W^{\prime}= \begin{cases}A W-2 \operatorname{Re}\left\{\bar{q}^{*}(0) f_{0} q(\theta)\right\}, & -\tau_{k}^{(j)} \leq \theta<0, \\
A W-2 \operatorname{Re}\left\{\bar{q}^{*}(0) f_{0} q(\theta)\right\}+f_{0}, & \theta=0\end{cases} \\
& \stackrel{\text { def }}{=} A W+H(z, \bar{z}, \theta),
\end{aligned}
$$

where

$$
H(z, \bar{z}, \theta)=H_{20}(\theta) \frac{z^{2}}{2}+H_{11}(\theta) z \bar{z}+H_{02}(\theta) \frac{\bar{z}^{2}}{2}+\cdots
$$

Comparing the coefficients, we obtain

$$
\begin{aligned}
& \left(A-2 i \omega_{0}\right) W_{20}(\theta)=-H_{20}(\theta), \\
& A W_{11}(\theta)=-H_{11}(\theta)
\end{aligned}
$$

And we know that for $\theta \in\left[-\tau_{k}^{(j)}, 0\right)$,

$$
H(z, \bar{z}, \theta)=-\bar{q}^{*}(0) f_{0} q(\theta)-q^{*}(0) \bar{f}_{0} \bar{q}(\theta)=-g(z, \bar{z}) q(\theta)-\bar{g}(z, \bar{z}) \bar{q}(\theta)
$$


Comparing the coefficients of (3.25) with (3.28) gives

$$
\begin{aligned}
& H_{20}(\theta)=-g_{20} q(\theta)-\bar{g}_{02} \bar{q}(\theta), \\
& H_{11}(\theta)=-g_{11} q(\theta)-\bar{g}_{11} \bar{q}(\theta) .
\end{aligned}
$$

From (3.26), (3.29), and the definition of $A$, we get

$$
\dot{W}_{20}(\theta)=2 i \omega_{k} W_{20}(\theta)+g_{20} q(\theta)+\bar{g}_{02} \bar{q}(\theta) \text {. }
$$

Noting that $q(\theta)=q(0) e^{i \omega_{0} \theta}$, we have

$$
W_{20}(\theta)=\frac{i g_{20}}{\omega_{k}} q(0) e^{i \omega_{k} \theta}+\frac{i \bar{g}_{02}}{3 \omega_{k}} \bar{q}(0) e^{-i \omega_{k} \theta}+E_{1} e^{2 i \omega_{k} \theta}
$$

where $E_{1}$ is a constant vector. Similarly, from (3.27), (3.30), and the definition of $A$, we have

$$
\begin{aligned}
& \dot{W}_{11}(\theta)=g_{11} q(\theta)+\bar{g}_{11} \bar{q}(\theta), \\
& W_{11}(\theta)=-\frac{i g_{11}}{\omega_{k}} q(0) e^{i \omega_{k} \theta}+\frac{i \bar{g}_{11}}{\omega_{k}} \bar{q}(0) e^{-i \omega_{k} \theta}+E_{2},
\end{aligned}
$$

where $E_{2}$ is a constant vector.

In the following, we shall seek appropriate $E_{1}, E_{2}$ in (3.32), (3.34), respectively. It follows from the definition of $A$ and (3.29), (3.30) that

$$
\int_{-\tau_{k}^{(j)}}^{0} d \eta(\theta) W_{20}(\theta)=2 i \omega_{k} W_{20}(0)-H_{20}(0)
$$

and

$$
\int_{-\tau_{k}^{(j)}}^{0} d \eta(\theta) W_{11}(\theta)=-H_{11}(0),
$$

where $\eta(\theta)=\eta(0, \theta)$.

From (3.30), we have

$$
H_{20}(0)=-g_{20} q(0)-\bar{g}_{02} \bar{q}(0)+\left(H_{1}, H_{2}, H_{3}, H_{4}\right)^{T},
$$

where

$$
\begin{aligned}
& H_{1}=2\left(a_{3}+a_{4} e^{-i \omega_{k} \tau_{k}^{(j)}}+a_{5} r_{3}+a_{6} r_{3} e^{-i \omega_{k} \tau_{k}^{(j)}}\right), \\
& H_{2}=2\left(b_{3} r_{1}^{2}+b_{4} r_{1}^{2} e^{-i \omega_{k} \tau_{k}^{(j)}}+b_{5} r_{1} r_{3}+b_{6} r_{1} r_{3} e^{-i \omega_{k} \tau_{k}^{(j)}}\right), \\
& H_{3}=2\left(c_{3} r_{2}^{2}+c_{4} r_{2}^{2} e^{-i \omega_{k} \tau_{k}^{(j)}}+c_{5} r_{2} r_{3}+c_{6} r_{2} r_{3} e^{-i \omega_{k} \tau_{k}^{(j)}}\right), \\
& H_{4}=2\left(d_{1} r_{3} e^{-i \omega_{k} \tau_{k}^{(j)}}+d_{1} r_{1} r_{3} e^{-i \omega_{k} \tau_{k}^{(j)}}+d_{1} r_{2} r_{3} e^{-i \omega_{k} \tau_{k}^{(j)}}\right),
\end{aligned}
$$


From (3.31), we have

$$
H_{11}(0)=-g_{11} q(0)-\bar{g}_{11}(0) \bar{q}(0)+\left(P_{1}, P_{2}, P_{3}, P_{4}\right)^{T},
$$

where

$$
\begin{aligned}
& P_{1}=2 a_{3}+2 a_{4} \operatorname{Re}\left\{r_{3} e^{i \omega_{k} \tau_{k}^{(j)}}\right\}+2 a_{5} \operatorname{Re}\left\{r_{3}\right\}+2 a_{6} \operatorname{Re}\left\{r_{3} e^{i \omega_{k} \tau_{k}^{(j)}}\right\} \\
& P_{2}=2 b_{3}\left|r_{1}\right|^{2}+2 b_{4} \operatorname{Re}\left\{r_{1} \bar{r}_{1} e^{-i \omega_{k} \tau_{k}^{(j)}}\right\}+2 b_{5} \operatorname{Re}\left\{r_{1} \bar{r}_{3}\right\}+2 b_{6} \operatorname{Re}\left\{r_{1} \bar{r}_{3} e^{-i \omega_{k} \tau_{k}^{(j)}}\right\}, \\
& P_{3}=2 c_{3}\left|r_{2}\right|^{2}+2 c_{4} \operatorname{Re}\left\{\left|r_{2}\right|^{2} e^{i \omega_{k} \tau_{k}^{(j)}}\right\}+2 c_{5} \operatorname{Re}\left\{r_{2} \bar{r}_{3}\right\}+2 c_{6} \operatorname{Re}\left\{r_{2} \bar{r}_{3} e^{-i \omega_{k} \tau_{k}^{(j)}}\right\}, \\
& P_{4}=2 d_{1} \operatorname{Re}\left\{r_{3} e^{i \omega_{k} \tau_{k}^{(j)}}\right\}+2 d_{1} \operatorname{Re}\left\{r_{1} e^{-i \omega_{k} \tau_{k}^{(j)}}\right\}+2 d_{1} \operatorname{Re}\left\{r_{2} e^{-i \omega_{k} \tau_{k}^{(j)}}\right\} .
\end{aligned}
$$

From (3.26), (3.27), and the definition of $A$, we have

$$
\left\{\begin{array}{l}
B W_{20}(0)+B_{1} W_{20}\left(-\tau_{k}^{(j)}\right)=2 i \omega_{k} W_{20}(0)-H_{20}(0) \\
B W_{11}(0)+B_{1} W_{11}\left(-\tau_{k}^{(j)}\right)=-H_{11}(0)
\end{array}\right.
$$

Noting that

$$
\begin{aligned}
& \left(i \omega_{k} I-\int_{-\tau_{k}^{(j)}}^{0} e^{i \omega_{k} \theta} d \eta(\theta)\right) q(0)=0, \\
& \left(-i \omega_{k} I-\int_{-\tau_{k}^{(j)}}^{0} e^{-i \omega_{k} \theta} d \eta(\theta)\right) \bar{q}(0)=0,
\end{aligned}
$$

and substituting (3.36) and (3.41) into (3.39), we have

$$
\left(2 i \omega_{k} I-\int_{-\tau_{k}^{(j)}}^{0} e^{2 i \omega_{k} \theta} d \eta(\theta)\right) E_{1}=\left(H_{1}, H_{2}, H_{3}, H_{4}\right)^{T}
$$

That is,

$$
\operatorname{det}\left(\begin{array}{cccc}
2 i \omega_{k}-a_{1} & 0 & 0 & -a_{2} \\
0 & 2 i \omega_{k}-b_{1} & 0 & -b_{2} \\
0 & 0 & 2 i \omega_{k}-c_{1} & -c_{2} \\
-d_{1} e^{-2 i \omega_{k} \tau_{k}^{(j)}} & -d_{1} e^{-2 i \omega_{k} \tau_{k}^{(j)}} & -d_{1} e^{-2 i \omega_{k} \tau_{k}^{(j)}} & 2 i \omega_{k}
\end{array}\right)\left(\begin{array}{l}
E_{1}^{(1)} \\
E_{1}^{(2)} \\
E_{1}^{(3)} \\
E_{1}^{(4)}
\end{array}\right)=\left(\begin{array}{l}
H_{1} \\
H_{2} \\
H_{3} \\
H_{4}
\end{array}\right)
$$

Hence,

$$
E_{1}^{(1)}=\frac{\Delta_{11}}{\Delta_{1}}, \quad E_{1}^{(2)}=\frac{\Delta_{12}}{\Delta_{1}}, \quad E_{1}^{(3)}=\frac{\Delta_{13}}{\Delta_{1}}, \quad E_{1}^{(4)}=\frac{\Delta_{14}}{\Delta_{1}},
$$

where

$$
\Delta_{1}=\operatorname{det}\left(\begin{array}{cccc}
2 i \omega_{k}-a_{1} & 0 & 0 & -a_{2} \\
0 & 2 i \omega_{k}-b_{1} & 0 & -b_{2} \\
0 & 0 & 2 i \omega_{k}-c_{1} & -c_{2} \\
-d_{1} e^{-2 i \omega_{k} \tau_{k}^{(j)}} & -d_{1} e^{-2 i \omega_{k} \tau_{k}^{(j)}} & -d_{1} e^{-2 i \omega_{k} \tau_{k}^{(j)}} & 2 i \omega_{k}
\end{array}\right)
$$




$$
\begin{aligned}
& \Delta_{11}=\operatorname{det}\left(\begin{array}{cccc}
H_{1} & 0 & 0 & -a_{2} \\
H_{2} & 2 i \omega_{k}-b_{1} & 0 & -b_{2} \\
H_{3} 1 & 0 & 2 i \omega_{k}-c_{1} & -c_{2} \\
H_{4} & -d_{1} e^{-2 i \omega_{k} \tau_{k}^{(j)}} & -d_{1} e^{-2 i \omega_{k} \tau_{k}^{(j)}} & 2 i \omega_{k}
\end{array}\right), \\
& \Delta_{12}=\operatorname{det}\left(\begin{array}{cccc}
2 i \omega_{k}-a_{1} & H_{1} & 0 & -a_{2} \\
0 & H_{2} & 0 & -b_{2} \\
0 & H_{3} & 2 i \omega_{k}-c_{1} & -c_{2} \\
-d_{1} e^{-2 i \omega_{k} \tau_{k}^{(j)}} & H_{4} & -d_{1} e^{-2 i \omega_{k} \tau_{k}^{(j)}} & 2 i \omega_{k}
\end{array}\right), \\
& \Delta_{13}=\operatorname{det}\left(\begin{array}{cccc}
2 i \omega_{k}-a_{1} & 0 & H_{1} & -a_{2} \\
0 & 2 i \omega_{k}-b_{1} & H_{2} & -b_{2} \\
0 & 0 & H_{3} & -c_{2} \\
-d_{1} e^{-2 i \omega_{k} \tau_{k}^{(j)}} & -d_{1} e^{-2 i \omega_{k} \tau_{k}^{(j)}} & H_{4} & 2 i \omega_{k}
\end{array}\right), \\
& \Delta_{14}=\operatorname{det}\left(\begin{array}{cccc}
2 i \omega_{k}-a_{1} & 0 & & 0 \\
0 & 2 i \omega_{k}-b_{1} & 0 & H_{1} \\
0 & 0 & 2 i \omega_{k}-c_{1} & H_{3} \\
-d_{1} e^{-2 i \omega_{k} \tau_{k}^{(j)}} & -d_{1} e^{-2 i \omega_{k} \tau_{k}^{(j)}} & -d_{1} e^{-2 i \omega_{k} \tau_{k}^{(j)}} & H_{4}
\end{array}\right) .
\end{aligned}
$$

Similarly, substituting (3.37) and (3.42) into (3.40), we have

$$
\left(\int_{-\tau_{k}^{(j)}}^{0} d \eta(\theta)\right) E_{2}=\left(P_{1}, P_{2}, P_{3}, P_{4}\right)^{T}
$$

That is,

$$
\left(\begin{array}{cccc}
a_{1} & 0 & 0 & a_{2} \\
0 & b_{1} & 0 & b_{2} \\
0 & 0 & c_{1} & c_{2} \\
d_{1} & d_{1} & d_{1} & 0
\end{array}\right)\left(\begin{array}{l}
E_{2}^{(1)} \\
E_{2}^{(2)} \\
E_{2}^{(3)} \\
E_{2}^{(4)}
\end{array}\right)=\left(\begin{array}{l}
P_{1} \\
P_{2} \\
P_{3} \\
P_{4}
\end{array}\right)
$$

Hence,

$$
E_{2}^{(1)}=\frac{\Delta_{21}}{\Delta_{2}}, \quad E_{2}^{(2)}=\frac{\Delta_{22}}{\Delta_{2}}, \quad E_{2}^{(3)}=\frac{\Delta_{23}}{\Delta_{2}}, \quad E_{2}^{(4)}=\frac{\Delta_{24}}{\Delta_{2}},
$$

where

$$
\begin{aligned}
& \Delta_{2}=\operatorname{det}\left(\begin{array}{cccc}
a_{1} & 0 & 0 & a_{2} \\
0 & b_{1} & 0 & b_{2} \\
0 & 0 & c_{1} & c_{2} \\
d_{1} & d_{1} & d_{1} & 0
\end{array}\right), \\
& \Delta_{21}=\operatorname{det}\left(\begin{array}{cccc}
-P_{1} & 0 & 0 & a_{2} \\
-P_{2} & b_{1} & 0 & b_{2} \\
-P_{3} & 0 & c_{1} & c_{2} \\
-P_{4} & d_{1} & d_{1} & 0
\end{array}\right),
\end{aligned}
$$




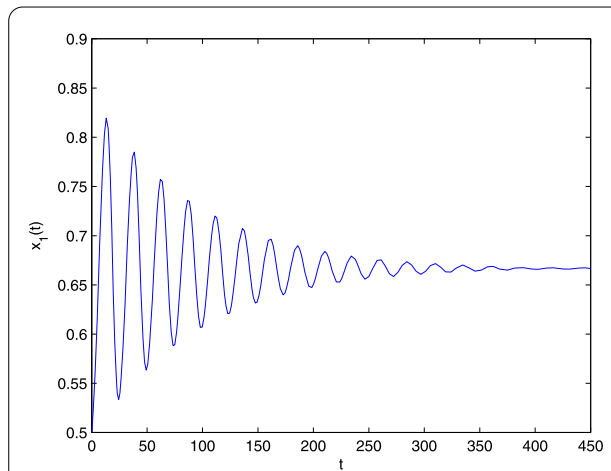

(a)

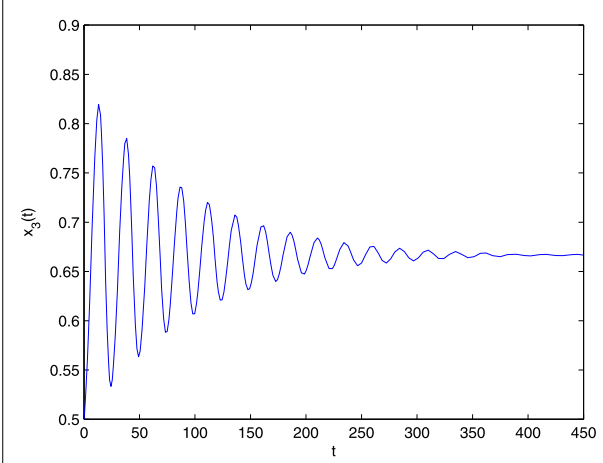

(c)

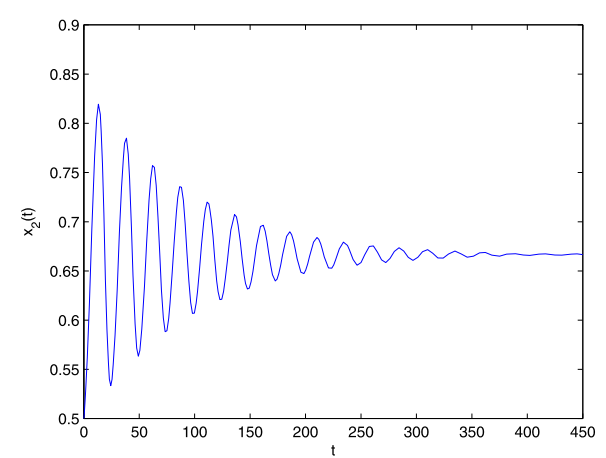

(b)

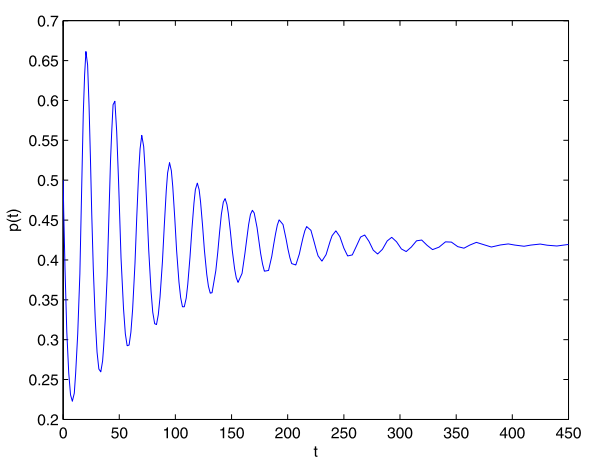

(d)

Figure 1 Dynamic behavior of system (4.1): times series of $x_{i}(i=1,2,3)$ and $p(t)$. A Matlab simulation of the asymptotically stable origin to system (4.1) with $\tau=1.65<\tau_{0} \approx 1.85$. The initial value is $(0.5,0.5,0.5,0.5)$.

$$
\begin{gathered}
\Delta_{22}=\operatorname{det}\left(\begin{array}{cccc}
a_{1} & -P_{1} & 0 & a_{2} \\
0 & -P_{2} & 0 & b_{2} \\
0 & -P_{3} & c_{1} & c_{2} \\
d_{1} & -P_{4} & d_{1} & 0
\end{array}\right), \\
\Delta_{23}=\operatorname{det}\left(\begin{array}{cccc}
a_{1} & 0 & -P_{1} & a_{2} \\
0 & b_{1} & -P_{2} & b_{2} \\
0 & 0 & -P_{3} & c_{2} \\
d_{1} & d_{1} & -P_{4} & 0
\end{array}\right), \\
\Delta_{24}=\operatorname{det}\left(\begin{array}{cccc}
a_{1} & 0 & 0 & -P_{1} \\
0 & b_{1} & 0 & -P_{2} \\
0 & 0 & c_{1} & -P_{3} \\
d_{1} & d_{1} & d_{1} & -P_{4}
\end{array}\right) .
\end{gathered}
$$

From (3.32), (3.34), (3.44), (3.47), we can calculate $g_{21}$ and derive the following values:

$$
\begin{aligned}
& c_{1}(0)=\frac{i}{2 \omega_{k}}\left(g_{20} g_{11}-2\left|g_{11}\right|^{2}-\frac{\left|g_{02}\right|^{2}}{3}\right)+\frac{g_{21}}{2}, \\
& \mu_{2}=-\frac{\operatorname{Re}\left\{c_{1}(0)\right\}}{\operatorname{Re}\left\{\lambda^{\prime}\left(\tau_{k}^{(j)}\right)\right\}},
\end{aligned}
$$




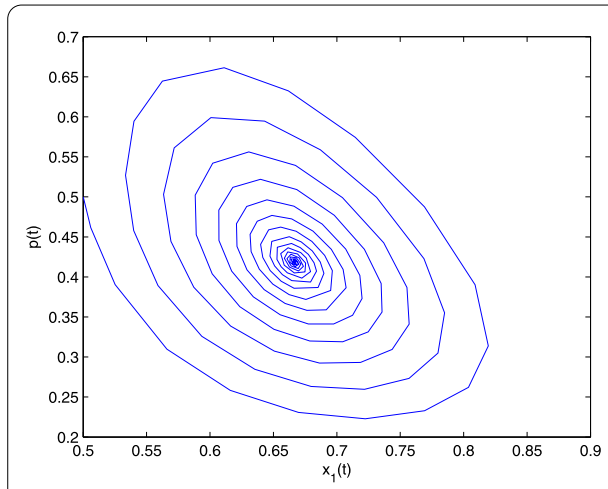

(a)

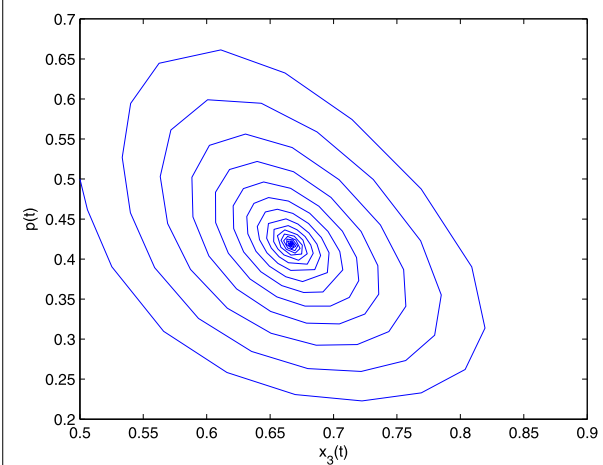

(c)

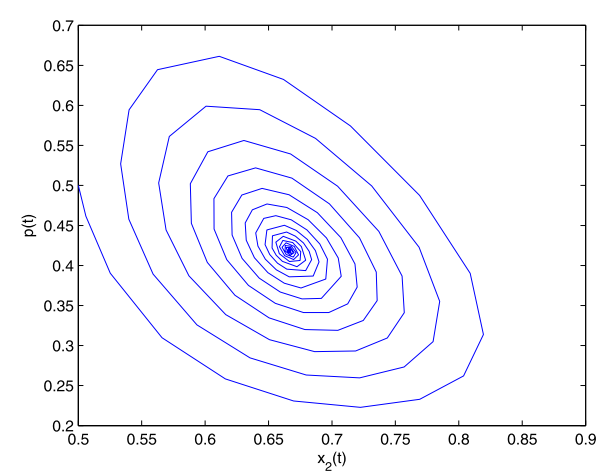

(b)

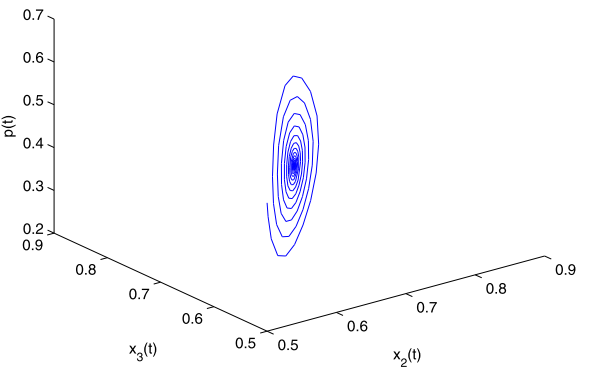

(d)

Figure 2 Dynamic behavior of system (4.1): projection on $x_{1}-p, x_{2}-p, x_{3}-p$ plane and projection on $\boldsymbol{x}_{\mathbf{2}}-\boldsymbol{x}_{\mathbf{3}} \boldsymbol{-} \boldsymbol{p}$ space. A Matlab simulation of the asymptotically stable origin to system (4.1) with $\tau=1.65<\tau_{0} \approx 1.85$. The initial value is $(0.5,0.5,0.5,0.5)$.

$$
\begin{aligned}
& \beta_{2}=2 \operatorname{Re}\left(c_{1}(0)\right), \\
& T_{2}=-\frac{\operatorname{Im}\left\{c_{1}(0)\right\}+\mu_{2} \operatorname{Im}\left\{\lambda^{\prime}\left(\tau_{k}^{(j)}\right)\right\}}{\omega_{k}} .
\end{aligned}
$$

These formulas give a description of the Hopf bifurcation periodic solutions of (1.4) at $\tau=\tau_{k}^{(j)}$ on the center manifold. From the discussion above, we have the following result.

Theorem 3.3 For system (1.4), if (H1)-(H4) hold, the periodic solution is supercritical (subcritical) if $\mu_{2}>0\left(\mu_{2}<0\right)$; the bifurcating periodic solutions are orbitally asymptotically stable with asymptotical phase (unstable) if $\beta_{2}<0\left(\beta_{2}>0\right)$; the periods of the bifurcating periodic solutions increase (decrease) if $T_{2}>0\left(T_{2}<0\right)$.

\section{Numerical examples}

In this section, we present some numerical results of system (1.4) to verify the analytical predictions obtained in the previous section. From Section 3, we may determine the direction of a Hopf bifurcation and the stability of the bifurcation periodic solutions. Let us 


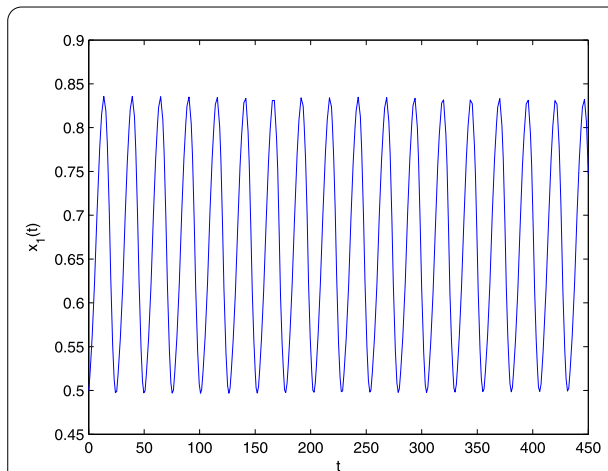

(a)

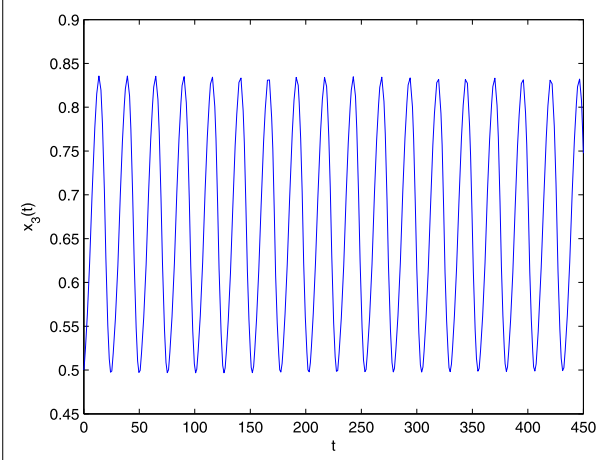

(c)

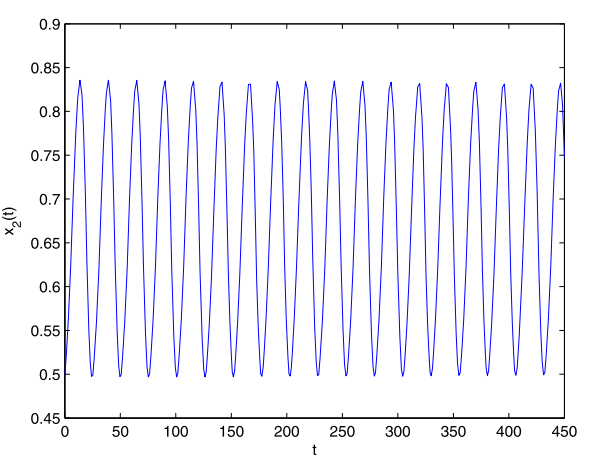

(b)

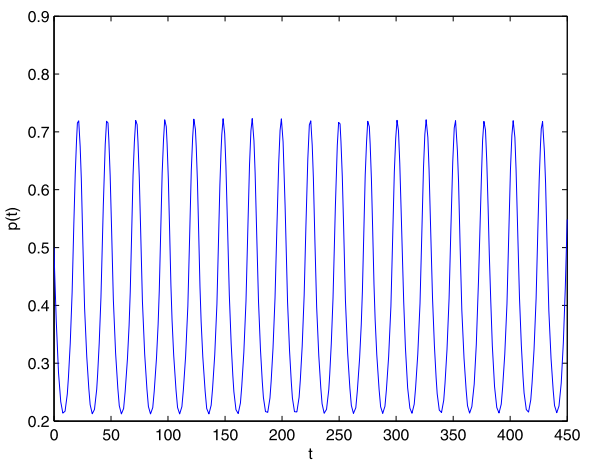

(d)

Figure 3 Dynamic behavior of system (4.1): times series of $\boldsymbol{x}_{\boldsymbol{i}}(\boldsymbol{i}=\mathbf{1 , 2 , 3 )}$ and $\boldsymbol{p}$. A Matlab simulation of a periodic solution to system (4.1) with $\tau=1.97>\tau_{0} \approx 1.85$. The initial value is $(0.5,0.5,0.5,0.5)$.

consider the following special case of system (1.4):

$$
\left\{\begin{array}{l}
\dot{x}_{1}(t)=0.5 x_{1}(t-\tau)\left[\frac{1-p(t)}{6.25 x_{1}(t)}-0.5 x_{1}(t) p(t)\right], \\
\dot{x}_{2}(t)=0.5 x_{2}(t-\tau)\left[\frac{1-p(t)}{6.25 x_{2}(t)}-0.5 x_{2}(t) p(t)\right], \\
\dot{x}_{3}(t)=0.5 x_{3}(t-\tau)\left[\frac{1-p(t)}{6.25 x_{3}(t)}-0.5 x_{3}(t) p(t)\right], \\
\dot{p}(t)=0.3 p(t)\left[x_{1}(t-\tau)+x_{2}(t-\tau)+x_{3}(t-\tau)-2\right] .
\end{array}\right.
$$

By some complicated computation by means of Matlab 7.0, we get $\omega_{0} \approx 0.9824, \tau_{0} \approx 1.85$, $\lambda^{\prime}\left(\tau_{0}\right) \approx 2.1022-3.1513 i$. Thus we can calculate the following values: $c_{1}(0) \approx-2.9542-$ $22.2355 i, \mu_{2} \approx 0.5642, \beta_{2} \approx-4.4636, T_{2} \approx 22.1327$. We see that the conditions indicated in Theorem 2.3 are satisfied. Furthermore, it follows that $\mu_{2}>0$ and $\beta_{2}<0$. Choose $\tau=1.65<\tau_{0} \approx 1.85$. Thus, the equilibrium $\left(x_{1}^{*}, x_{2}^{*}, x_{3}^{*}, p^{*}\right)$ is stable when $\tau<\tau_{0}$, which is illustrated by the computer simulations (see Figure 1 and Figure 2). When $\tau$ passes through the critical value $\tau_{0} \approx 6.2$, the equilibrium $\left(x_{1}^{*}, x_{2}^{*}, x_{3}^{*}, p^{*}\right)$ loses its stability and a Hopf bifurcation occurs, i.e., a family of periodic solutions bifurcate from the equilibrium $\left(x_{1}^{*}, x_{2}^{*}, x_{3}^{*}, p^{*}\right)$. Choose $\tau=1.97>\tau_{0} \approx 1.85$. Since $\mu_{2}>0$ and $\beta_{2}<0$, the direction of the Hopf bifurcation is $\tau>\tau_{0}$, and these bifurcating periodic solutions from $\left(x_{1}^{*}, x_{2}^{*}, x_{3}^{*}, p^{*}\right)$ at $\tau_{0}$ are stable; they are depicted in Figure 3 and Figure 4. 


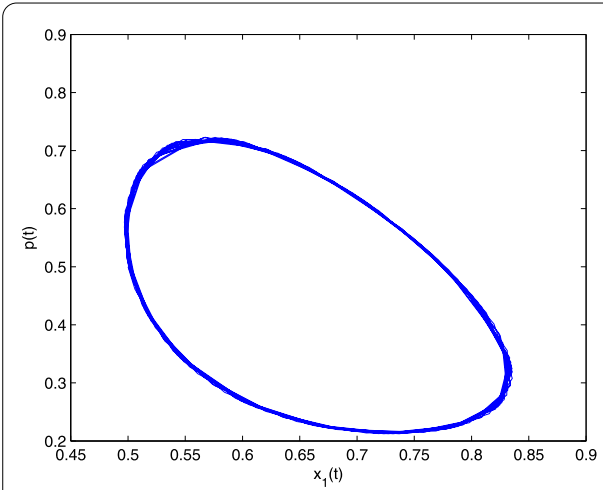

(a)

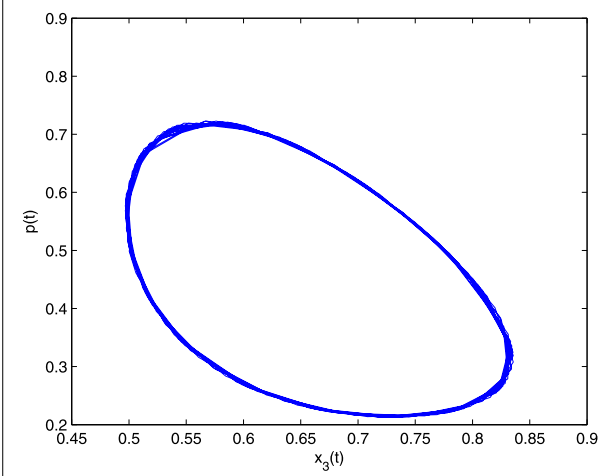

(c)

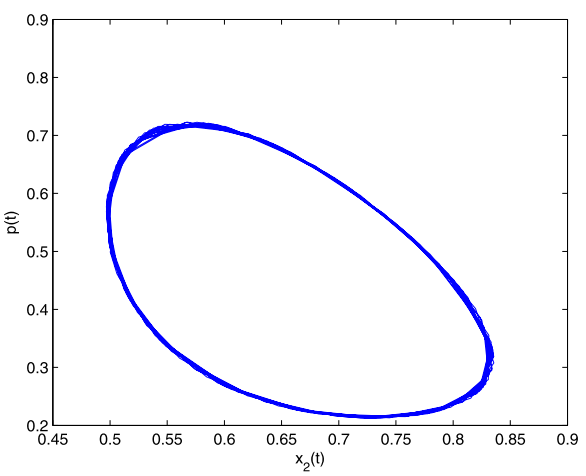

(b)

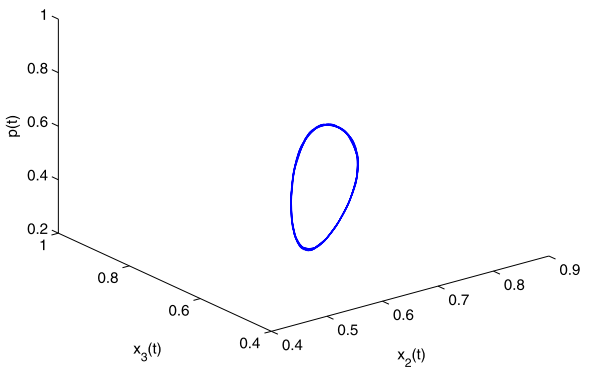

(d)

Figure 4 Dynamic behavior of system (4.1): projection on the $x_{1}-p, x_{2}-p, x_{3}-p$ plane and projection on the $x_{2}-x_{3}-p$ space, respectively. A Matlab simulation of a periodic solution to system (4.1) with $\tau=1.97>\tau_{0} \approx 1.85$. The initial value is $(0.5,0.5,0.5,0.5)$.

\section{Conclusions}

In this paper, we have investigated the properties of Hopf bifurcation in an exponential RED algorithm with communication delay. It is shown that under certain conditions, the Hopf bifurcation occurs as the delay $\tau$ passes through some critical values $\tau=\tau_{k}^{(j)}$, $k, j=0,1,2, \ldots$. Moreover, the direction of the Hopf bifurcation and the stability of the bifurcating periodic orbits are derived by applying the normal form theory and the center manifold theorem.

Competing interests

The authors declare that they have no competing interests.

\section{Authors' contributions}

The authors have made contributions of the same significance. All authors read and approved the final manuscript.

\section{Author details}

'Guizhou Key Laboratory of Economics System Simulation, Guizhou University of Finance and Economics, Guiyang, 550004, P.R. China. '2School of Mathematics and Statistics, Henan University of Science and Technology, Luoyang, 471023, P.R. China.

\section{Acknowledgements}

The first author was supported by National Natural Science Foundation of China (No. 11261010), Natural Science and Technology Foundation of Guizhou Province (J[2015]2025) and 125 Special Major Science and Technology of Department of Education of Guizhou Province ([2012]011). The second author was supported by National Natural 
Science Foundation of China (No. 11101126). The authors would like to thank the referees and the editor for helpful suggestions incorporated into this paper.

Received: 10 October 2015 Accepted: 28 November 2015 Published online: 03 February 2016

\section{References}

1. Liu, F, Guan, ZH, Wang, HO: Controlling bifurcations and chaos in TCP-UDP-RED. Nonlinear Anal., Real World Appl. 11(3), 1491-1501 (2010)

2. Raina, G, Heckmann, O: TCP: local stability and Hopf bifurcation. Perform. Eval. 64, 266-275 (2007)

3. Guo, ST, Liao, XF, Li, CD, Yang, DG: Stability analysis of a novel exponential-RED model with heterogeneous delays. Comput. Commun. 30, 1058-1074 (2007)

4. Xu, CJ, Tang, XH, Liao, MX: Local Hopf bifurcation and global existence of periodic solutions in TCP system. Appl. Math. Mech. 31(6), 775-786 (2010)

5. Guo, ST, Feng, G, Liao, XF, Liu, Q: Novel delay-range-dependent stability analysis of the second-order congestion control algorithm with heterogenous communication delays. J. Netw. Comput. Appl. 32, 568-577 (2009)

6. Tian, YP: A general stability criterion for congestion control with diverse communication delays. Automatica 41, 1255-1262 (2005)

7. Abdallah, CT, Chiasson, J, Tarbouriech, S (eds.): Advances in Communication Control Networks. Lecture Notes in Control and Information Sciences. Springer, Berlin (2004)

8. Srikant, R: The Mathematics of Internet Congestion Control. Birkhäuser, Boston (2004)

9. Liu, S, Basar, T, Srikant, R: Exponential-RED: a stabilizing AQM scheme for low-and high-speed TCP protocols. IEEE/ACM Trans. Netw. 13(5), 1068-1081 (2005)

10. Alpcan, T, Basar, T: A globally stable adaptive congestion control scheme for Internet-style networks with delays. IEEE/ACM Trans. Netw. 13(6), 1261-1274 (2005)

11. Raina, G: Local bifurcation analysis of some dual congestion control algorithms. IEEE Trans. Autom. Control 50(8), 1135-1146 (2005)

12. Sichitiu, ML, Bauter, PH: Asymptotic stability of congestion control systems with multiple sources. IEEE Trans. Autom. Control 51(2), 292-298 (2006)

13. Guo, ST, Liao, XF, Liu, Q, Li, CD: Necessary and sufficient conditions for Hopf bifurcation in exponential RED algorithm with communication delay. Nonlinear Anal., Real World Appl. 9(4), 1768-1793 (2008)

14. Ruan, $\mathrm{S}, \mathrm{Wei}, \mathrm{J}$ : On the zero of some transcendental functions with applications to stability of delay differential equations with two delays. Dyn. Contin. Discrete Impuls. Syst., Ser. A Math. Anal. 10, 863-874 (2003)

15. Hassard, B, Kazatina, D, Wan, Y: Theory and Applications of Hopf Bifurcation. Cambridge University Press, Cambridge (1981)

\section{Submit your manuscript to a SpringerOpen ${ }^{\ominus}$ journal and benefit from:}

- Convenient online submission

Rigorous peer review

- Immediate publication on acceptance

- Open access: articles freely available online

- High visibility within the field

- Retaining the copyright to your article 